\title{
Les Scaphidiidae (Coleoptera) de la Nouvelle-Calédonie
}

par

\author{
Ivan LÖBL
}

Avec 42 figures

\section{Abstract}

Scaphidiidae (Coleoptera) of New Caledonia. - Review of the New Caledonian members of the family Scaphidiidae based on a collection made in October 1978. Five genera and 34 species are now known from the island. New taxa are: Pseudobironium globosum sp. n., Baeocera dugdalei sp. n., Zinda gen. n. teres sp. n. and Z. abdita sp. n., Scaphisoma concolor sp. n., S. confrater sp. n., S. modestum sp. n., S. funebre sp. n., S. pallens sp. n., S. kuschelianum sp. n., S. excellens sp. n., S. variabile sp. n., plus one species of the genus Scaphisoma Leach left unnamed. Keys are given for genera and species.

\section{INTRODUCTION}

Les Scaphidiides de la Nouvelle-Calédonie ont été étudiés dans quelques notes que j'ai publiées au cours de ces dernières années. Le nombre des espèces connues a ainsi augmenté de 2 à 21, mais une étude d'ensemble faisait défaut. Récemment, mon collègue $\mathrm{D}^{\mathrm{r}} \mathrm{G}$. Kuschel, entomologiste au Department of Scientific and Industrial Research à Auckland, a effectué, avec ses collaborateurs MM. J. S. Dugdale et J. C. Watt, une mission en Nouvelle-Calédonie. L'ensemble de Scaphidiides recueillis comporte en plus de la plupart des formes déjà décrites 13 espèces nouvelles, dont deux appartiennent à un genre inédit. Ce brillant résultat, dû à la compétence du $\mathrm{D}^{\mathrm{r}}$ Kuschel qui a su méthodiquement exploiter les sites forestiers avec des techniques appropriées (tamisages de débris végétaux et extraction de la faune cryptique dans les appareils "Winkler»), nous donne une idée assez précise sur l'importance qualitative et quantitative de la famille sur cette île.

La faune de la Nouvelle-Calédonie présente, par rapport aux autres îles mélanésiennes, des particularités dues à l'ancienneté du peuplement et à son isolement. GressiTT (1961) sépare la Nouvelle-Calédonie avec les îles Norfolk et Lord Howe, de la Mélanésie orientale, dans une division biogéographique à part. D'après SolEm (1958) la Nouvelle- 
Calédonie aurait eu une ancienne liaison avec la Nouvelle-Guinée, indépendante et antérieure à celle des Nouvelles-Hébrides avec la Nouvelle-Guinée. Le fait le plus émergent des données actuellement disponibles est le haut degré d'endémisme et l'absence de groupes d'espèces ou de genres répandus sur les îles au nord-ouest de la Nouvelle-Calédonie. En ce qui concerne les Scaphidiides, sur les cinq genres présents, deux (Brachynoposoma Löbl et Zinda gen. n., ayant subi une évolution régressive) sont endémiques. Notons aussi que toutes les 34 espèces décrites à ce jour apparaissent endémiques; mais notre connaissance concernant la faune mélanésienne reste encore incomplète et il est bien possible que certaines, du genre Scaphisoma Leach notamment, ont une aire de répartition plus vaste. Remarquable est la découverte d'une espèce du genre Pseudobironium Pic, jusqu'ici inconnue au sud-est de la ligne Java-Bornéo-Philippines. Les espèces du genre Baeocera Erichson trouvées en Nouvelle-Calédonie forment un groupe isolé très distinct; les autres Baeocera mélanésiens appartiennent au groupe lenta (asiatique) ou semblent dérivés de ce groupe. Le genre Scaphisoma Leach est représenté en Nouvelle-Calédonie par 27 espèces, dont seulement une, pallens sp. n., semble apparentée aux espèces de la Nouvelle-Guinée et de l'Asie. Le groupe endémique reticulatum, avec 9 espèces, dont certaines apparemment fréquentes sur l'île, est particulièrement caractéristique. Le groupe tannaense des Nouvelles-Hébrides et de Fiji ainsi que le groupe haemorrhoidale, répandus de l'Asie tropicale à la Nouvelle-Guinée au sud et jusqu'au Samoa et Hawaii à l'est, y manquent. Les genres Scaphobaeocera Csiki et Scaphoxium Löbl font également défaut ${ }^{1}$. Ils peuplent pourtant l'est de l'Australie, la Nouvelle-Guinée et la Nouvelle-Irlande, Scaphobaeocera les îles Carolines, Scaphoxium les Nouvelles-Hébrides et Fiji.

Abréviations utilisées pour les collections étudiées:

\begin{tabular}{|c|c|c|}
\hline BISHOP & - & Bishop Museum, Honolulu. \\
\hline FMNH & - & Field Museum of Natural History, Chicago. \\
\hline FRANZ & & Collection du professeur H. Franz, Mödling bei Wien. \\
\hline IRSN & & Institut Royal des Sciences naturelles, Bruxelles. \\
\hline MG & & Muséum d’histoire naturelle, Genève. \\
\hline NZAC & & New Zealand Arthropod Collection, Auckland. \\
\hline MB & & Természettudomanyi Muzeum, Budapest. \\
\hline
\end{tabular}

Remarques. La longueur du corps est mesurée du bord apical du pronotum aux angles apicaux internes des élytres; uniquement les parties découvertes des mésépimères et métépisternes sont mesurées.

\section{SYSTÉMATIQUE}

\section{TABLEAU DES GENRES}

1 Angles postérieurs du pronotum prolongés en arrière, situés derrière le niveau du bord antérieur des métépisternes

${ }^{1}$ L'exemplaire identifié « Scaphobaeocera sp. indet. » et signalé du Mt. Koghis (LöBL, 1973) était pourvu d'une fausse étiquette de localité. Il appartient à franzi Löbl du Queensland (voir LöBL, 1977a). 
- Angles postérieurs du pronotum pas prolongés, situés devant le niveau du bord antérieur des métépisternes . . . . . . . . . . Pseudobironium

2 Forme générale naviculaire ou ovale. Pronotum avec un lobe basal plus ou moins développé

- Forme générale globulaire. Lobe basal du pronotum non développé .

3 Article antennaire III allongé. Pas d'aires postcoxales sur le premier sternite apparent

- Article antennaire III court, asymétrique, triangulaire. Premier sternite apparent avec des aires postcoxales . . . . . . . . . . Scaphisoma

4 Elytres réunis pas plus larges que le pronotum, non rétrécis vers la base. Espèces de taille minuscule, microphtalmes et aptères . . . . . Zinda

- Elytres réunis plus larges que le pronotum, plus ou moins rétrécis vers la base. Espèces de taille petite ou moyenne, exceptionnellement moins longues de $1 \mathrm{~mm}$, macrophtalmes et généralement ailées . . . . . . . Baeocera

\section{Pseudobironium Pic}

Ce genre renferme une vingtaine d'espèces répandues dans la région orientale, au Japon et dans l'extrême est d'URSS, vers le sud-est jusqu'aux Philippines, à Bornéo et à Java. La découverte d'une nouvelle espèce en Nouvelle-Calédonie était donc inattendue.

\section{Pseudobironium globosum sp. n.}

Holotype đ: Col d'Amieu 550 m, 15.X.1978, G. Kuschel, tamisage de débris végétaux et de bois pourri sur une crête; NZAC.

Paratypes: comme holotype, 2 oे 2 \% ; NZAC, MG.

Longueur 1,8-1,9 mm. Corps convexe, entièrement d'un brun rougeâtre clair. Apex de l'abdomen, tarses, articles antennaires I et II et la base des articles III à VI encore plus clairs à ocres, la partie distale des articles III à VI et les articles suivants d'un brun foncé. Articles III des palpes maxillaires à l'apex nettement plus large que la base de l'article IV. Rapport des longueurs des articles antennaires: III $7:$ IV $9:$ V 10 : VI 8 : VII 18 : VIII 12 : IX $20:$ X $20:$ XI 27 (holotype); articles III et IV très grêles, de même largeur, progressivement et légèrement plus épais vers l'apex; V et VI seulement un peu plus épais; VII 3 fois plus long que large; VIII presque 3 fois plus long que large, un peu plus large que VI; IX et X nettement plus larges que VII, XI encore plus large, à peu près 2 fois plus long que large. Pronotum à la base de $1,15-1,25 \mathrm{~mm}$, sa ponctuation assez dense et très fine, à peine visible au grossissement x 25 . Scutellum recouvert par le lobe basal du pronotum. Elytres longs sur la suture de 1,22-1,32 mm, larges de 1,24$1,40 \mathrm{~mm}$; côtés arrondis; disque légèrement aplati devant le bord apical; stries suturales fines; stries basales très fines et superficielles, entières ou effacées au milieu de la base; espace entre la suture et la strie suturale plat dans la moitié basale, un peu bombé dans la moitié apicale; ponctuation assez dense, nettement plus grosse que celle du pronotum mais très superficielle et fine. Pygidium orné d'une microsculpture peu marquée formée de points, sa ponctuation éparse et très fine. Ponctuation de la face ventrale partout éparse et extrêmement fine, à peine visible au grossissement $\times 100$. Métasternum légèrement 
convexe au milieu. Aires derrière les cavités coxales II longues de 0,05-0,06 mm, avec une rangée éparse de points marginaux relativement fins. Sternites avec une microsculpture formée de points particulièrement bien marqués sur les côtés du premier segment apparent. Celui-ci orné de chaque côté d'une petite dépression superficielle, tangente à la carène latérale. Tibias antérieurs droits, intermédiaires et postérieurs courbés, les postérieurs longs de 0,56-0,66 mm, munis d'un éperon apical seulement, leur face dorsale presque lisse. Tarses postérieurs nettement plus courts que les tibias, longs de $0,35-0,41 \mathrm{~mm}$, avec l'article I plus court que II et III réunis et plus court que V.

Mâle. Articles I à III des tarses antérieurs élargis. Edéage (fig. 1 et 2) long de 0,62$0,64 \mathrm{~mm}$.

Espèce remarquable par les tibias postérieurs muni d'un seul éperon apical, par les tarses courts et par le très long flagellum du sac interne de l'édéage. En outre, elle se distingue facilement par sa petite taille, sa coloration claire, sa ponctuation très fine et son scutellum recouvert.

\section{Baeocera Erichson}

Genre assez hétérogène, richement représenté dans les régions au climat chaud et humide mais largement répandu aussi dans les zones tempérées. En Nouvelle-Calédonie représenté par deux espèces très proches qui constituent un groupe isolé.

\section{TABLEAU DES BAEOCERA}

1 Premier sternite apparent avec une rangée basale de gros points plus ou moins allongés et souvent prolongés en arrière par des stries. Paramères de l'édéage larges et pubescents à l'apex . . . . . . . . . . . . . . . subaenea

- Gros points sur la base du premier sternite apparent non ou à peine allongés et non prolongés par des stries. Paramères de l'édéage rétrécis vers l'apex et dépourvus de pubescence dugdalei

\section{Baeocera subaenea (Fauvel)}

Scaphosoma subaeneum Fauvel, 1903: 292; holotype + : Boulari; IRSN.

Eubaeocera subaenea; LöBL 1969: 1, fig. 1 et 2.

Eubaeocera subaenea; LöBL 1973: 309, fig. 1 et 2.

Matériel étudié (33 ex.): le type unique de Boulari; Haute Vallée de la Houaillou et Gondé (LöBL 1973); 7 miles au sud-est de La Foa (LöBL 1974); La Crouen (LöBL 1977b); Mt. Dore, 8.X.1978, G. Kuschel, tamisage de feuilles mortes et de bois pourri dans un ravin, 1 ex.; Mt. Koghis 550 m, 7.X.1978, G. Kuschel, tamisage de feuilles mortes sur une pente, 1 ex.; Col de Mouirange, 11.X.1978, G. Kuschel, tamisage de feuilles mortes et de bois pourri au-dessous des buissons dans un ravin, 1 ex.; Rivière Thiem, 22 km au sud-est-sud d'Hienghène, 27.X.1978, G. Kuschel, tamisage de débris végétaux et de bois pourri dans la broussaille littorale, sol très sec, 1 ex.; Kavatch près d'Hienghène, 150 m, 28.X.1978, G. Kuschel, tamisage de débris végétaux et de bois pourri au fond d'un ravin près d'un ruisseau, 11 ex.; Mt. Panié $600 \mathrm{~m}, 28 . X .1978$, J. C. Watt, tamisage de feuilles mortes dans la forêt ombrophile, 2 ex.

Longueur 1,3-1,6 mm. Corps d'un brun très foncé à noir. Articles antennaires III à VI grêles, IV et VI à peu près aussi longs, V nettement plus long; articles suivants 

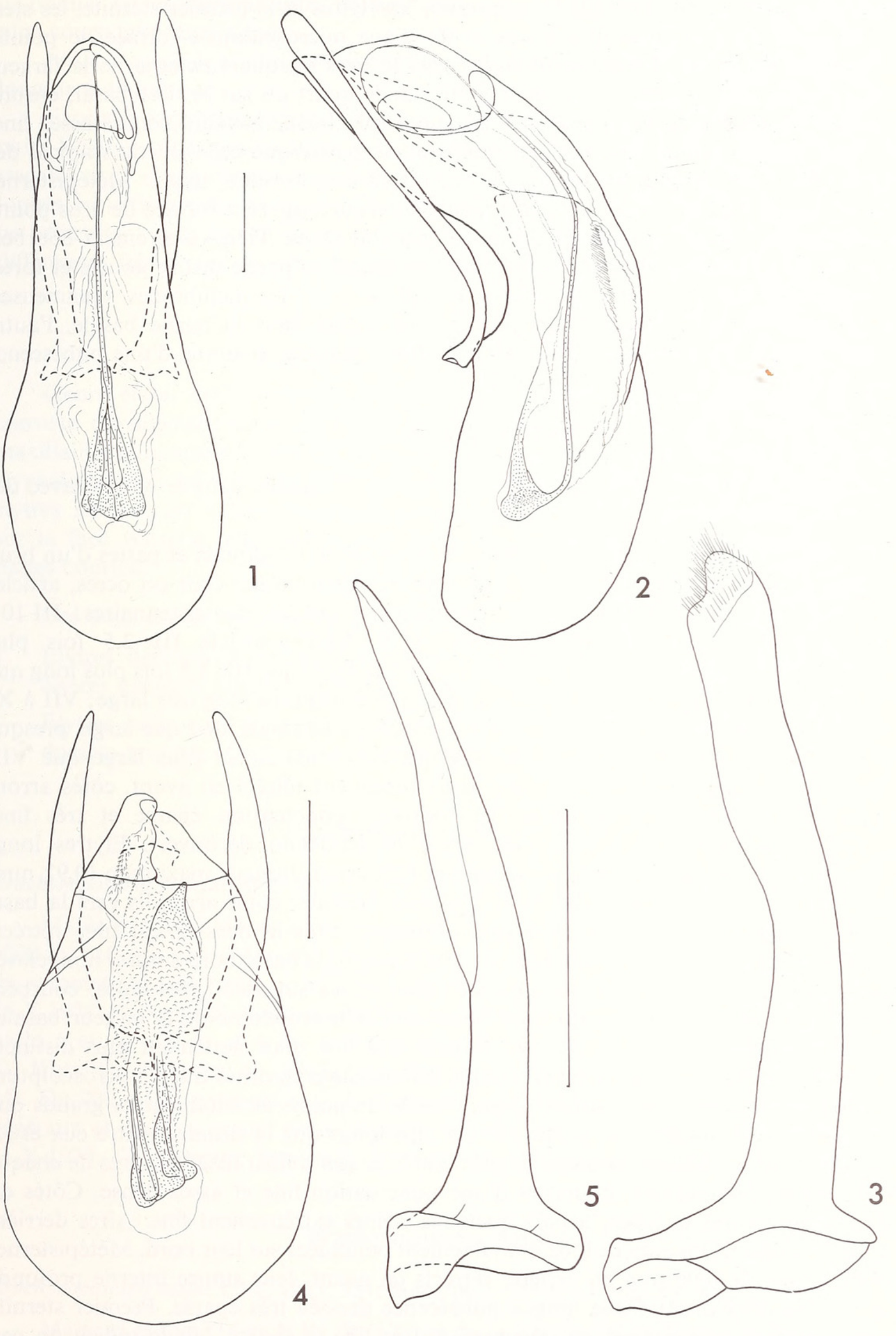

FIG. 1 à 5.

Edéages; 1 et 2. Pseudobironium globosum sp. n.; 3. Baeocera subaenea (Fauvel), paramère en vue ventrale; 4 et 5 . Baeocera dugdalei $\mathrm{sp}$. n., édéage (4) et paramère en vue ventrale (5), grossissement plus fort. Echelle $=0,1 \mathrm{~mm}$. 
larges. Pas de microsculpture sur le pronotum, les élytres et le premier sternite, les sternites suivants et les tergites découverts ornés d'une microsculpture formée de points. Elytres avec les stries suturales prolongées vers le tiers ou quart externe de la largeur basale; ponctuation discale éparse et très fine sur le quart ou sur le tiers basal, ensuite progressivement plus dense et grosse, sur le tiers apical généralement encore assez fine. Ponctuation du pygidium dense et au moins aussi grosse que celle du tiers apical des élytres. Mésépimères grands. Côtés du métasternum presque lisses. Métépisternes bombés. Rangée basale sur les côtés du premier sternite apparent formée de gros points plus ou moins allongés et souvent prolongés par des stries. Tibias légèrement courbés. Edéage long de 0,56-0,64 $\mathrm{mm}$. Lobe médian très grand, sa partie distale courte et fortement déclinée (vue latérale). Sac interne complexe, avec les membranes squameuses, orné de deux pièces chitinisées, une triangulaire située dans la partie basale, l'autre apicale allongée. Paramères élargis dans leur moitié apicale, pourvus d'une pubescence distale très courte (fig. 3 ).

\section{Baeocera dugdalei sp. $n$.}

Holotype ơ: Mt. Koghis 650 m, 9.X.1978, J. S. Dugdale, dans le terreau avec des hépatiques; NZAC.

Longueur 1,35 mm. Corps très bombé, noir, apex de l'abdomen et pattes d'un brun rougeâtre, articles antennaires I à VI d'un brun rougeâtre assez clair ou ocres, articles VII à XI d'un brun foncé. Rapport des longueurs des articles antennaires: III 10 : IV 10 : V 14 : VI 9 : VII 20 : VIII 17 : IX $21:$ X 19 :XI 27; article III 2,5 fois plus long que large; IV très légèrement plus grêle; V aussi large que III, 3,5 fois plus long que large; VI à peine plus large que $\mathrm{V}$, un peu plus que 2 fois plus long que large; VII à XI larges; VII presque 3 fois plus long que large; VIII 2,5 fois plus long que large, presque aussi large que VII; XI 2,3 fois plus long que large, nettement plus large que VII. Pronotum à la base large de $0,83 \mathrm{~mm}$, assez fortement rétréci en avant, côtés arrondis; carènes latérales pas visibles en vue dorsale; ponctuation éparse et très fine, à peine visible au grossissement $\times 25$. Apex du scutellum découvert. Elytres longs sur la suture de $0,86 \mathrm{~mm}$ (longueur maximum $0,98 \mathrm{~mm})$; largeur maximum $(0,92 \mathrm{~mm})$ située juste derrière le quart basal de la longueur latérale; côtés arrondis vers la base, presque rectilignes dans le tiers médian, rectilignes dans le tiers apical plus rétréci; carènes latérales visibles en vue dorsale seulement près de la base; bord sutural non relevé; espace entre la suture et les stries suturales plat; stries suturales profondes, courbées à la base et prolongées le long du bord basal vers le tiers externe de la largeur basale; ponctuation discale éparse et fine, sur la base très fine mais nettement plus distincte que celle du pronotum. Propygidium et pygidium découverts, ornés d'une microsculpture formée de points; leur ponctuation dense, formée de points au moins aussi grands que ceux des élytres. Mésépimères grands, 2 fois plus longs que la distance entre eux et les hanches II. Partie médiane du métasternum bombée, son milieu lisse, les aires de chaque côté du milieu pubescentes, pourvues d'une ponctuation fine et assez dense. Côtés du métasternum glabres et lisses, à part quelques points extrêmement fins. Aires derrière les cavités coxales II longues de $0,03 \mathrm{~mm}$, finement ponctuées sur leur bord. Métépisternes bombés, larges de $0,06 \mathrm{~mm}$ en arrière, rétrécis en avant, leur suture interne profonde et droite. Sternites ornés d'une longue pubescence dressée très éparse. Premier sternite apparent sans microsculpture, sa ponctuation très fine et éparse, sur le milieu un peu plus distincte que sur les côtés; rangée basale pas interrompue au milieu et ne dépassant pas latéralement le niveau de l'angle interne du métépimère, formée de gros points non ou très légèrement allongés. Sternites suivants ornés d'une microsculpture formée 
de points. Tibias assez robustes, légèrement courbés; tibias postérieurs longs de 0,42 mm, plus courts que leurs tarses.

Mâle. Article 1 à 3 des tarses antérieurs et intermédiaires élargis. Edéage (fig. 4 et 5) long de $0,44 \mathrm{~mm}$.

Espèce à caractères externes très similaires à subaenea; en diffère par l'article antennaire XI plus court et large (chez subaenea 2,6-3 fois plus long que large) et par le premier sternite orné d'une rangée basale de gros points non ou à peine allongés et non prolongés en arrière par des stries. Ces deux caractères, souvent variables chez Baeocera, ne permettent pas une distinction sûre. Par contre, l'édéage chez dugdalei montre des différences très nettes, au niveau des paramères et du sac interne en particulier.

\section{Brachynoposoma Löbl}

Genre établi pour deux espèces aptères caractérisées par le corps globuleux, très convexe dorsalement, assez plat sur la face ventrale, avec les antennes et les palpes maxillaires semblables à ceux de Baeocera, galea étroite, lacinia bien développée, suture clypéale effacée, lobe basal du pronotum réduit, ses angles postérieurs peu prolongés, élytres soudés, arrondis à l'apex, avec pseudopleures horizontaux situés entièrement sur la face ventrale, alinotum hypertrophié, mésépisternes grands, métasternum petit, pattes courtes.

Ces deux espèces peuvent être facilement distinguées:

1 Elytres dépourvus de microsculpture, ponctuation sur le pronotum et les élytres distincte au grossissement $\times 50$. . . . . . . . . . . . . . . punctatum

- Elytres avec une microsculpture bien marquée, ponctuation sur la face dorsale presque effacée, non ou à peine visible au grossissement $\times 100$. . major

\section{Brachynoposoma punctatum Löbl}

Brachynoposoma punctatum Löbl, 1973: 332, fig. 33-41; holotype đ̊: Table Unio; Franz.

Matériel étudié (19 ex.): les types de Table Unio et du Col de Hau; Table Unio $1000 \mathrm{~m}$, près du Col d'Amieu, 16.X.1978, G. Kuschel, tamisage de débris végétaux près du sommet sur une pente rocailleuse humide, 2 ex.; Table Unio près du Col d'Amieu, 16.X.1978, G. Kuschel, tamisage de débris végétaux et de bois pourri dans la forêt ombrophile 1 ex. à $900 \mathrm{~m}$ et 4 ex. à 800 d'altitude; Mt. Do, sommet $1020 \mathrm{~m}, 22 . X .1978$, G. Kuschel, tamisage de débris végétaux au-dessous de Araucaria biramulosa, 1 ex.; Mt. Do 900 m, au nord-ouest de Pouloupari, 22.X.1978, G. Kuschel, tamisage de débris végétaux et de bois pourri dans la forêt ombrophile, 4 ex.; Col des Roussettes $500 \mathrm{~m}$, 31.X.1978, G. Kuschel, tamisage de débris végétaux dans la forêt ombrophile, 1 ex.

\section{Brachynoposoma major Löbl}

Brachynoposoma major Löbl, 1973: 334; holotype $q$ : Rivière Bleue; Franz.

Matériel étudié (14 ex.): les deux types de la Rivière Bleue et du Pic du Pin; Rivière Bleue, 10.X.1978, G. Kuschel, tamisage de débris végétaux et de bois pourri à proximité de quelques Nothofagus codonandra, 2 ex.; Pic du Pin, 6.X.1978, G. Kuschel, tamisage de feuilles mortes dans un ravin avec Nothofagus, 1 ex.; Col de Mouirange, 11.X.1978, 
G. Kuschel, tamisage de feuilles mortes et de bois pourri au-dessous des buissons dans un ravin sec, 4 ex.; Mt. Koghis, Cascade 650 m, 12.X.1978, G. Kuschel, feuilles mortes dans un terrain marécageux avec fougères près d'un ruisseau, 1 ex.; Mt. Koghis $550 \mathrm{~m}$, 7.X.1978, G. Kuschel, tamisage de feuilles mortes sur une pente, 1 ex.; Mt. Ouénarou 300 m, 24.X.1978, G. Kuschel, tamisage de feuilles mortes et de bois pourri dans la forêt ombrophile, 2 ex.

Zinda gen. n.

Espèce-type: Zinda teres sp. $\mathrm{n}$.

Taille très petite. Corps ovale, face dorsale convexe, très bombée. Tête (fig. 6) assez grande. Yeux minuscules, situés chacun sur un petit tubercule, formés de quelques ommatidies plus ou moins dépigmentées. Aire antennaire concave limitée au-dessus et au-dessous par une carène. Insertion antennaire située devant l'œil. Suture du clypéus nette. Antennes avec les articles III à V allongés, VI et VIII minuscules, VII IX, X et XI larges. Mandibules (fig. 7) à deux pointes. Galea large, lacinia très grêle (fig. 8). Article III des palpes maxillaires robuste, IV fortement accuminé. Articles I et II des palpes labiaux transverses, III un peu plus long que large, IV grêle (fig. 9). Pronotum relativement très grand, en vue dorsale seulement un peu plus court que les élytres; bord basal sinué, lobe basal large et court, peu développé; bord antérieur convexe dans sa partie médiane; carène et strie latérales entières; face ventrale concave, profondément déprimée, bord basal de la face ventrale concave; angles postérieurs légèrement prolongés en arrière et situés au-dessus de la base des pseudopleures. Cavités coxales I fermées. Elytres pas plus larges que le pronotum, largeur maximum située à la base, vers l'apex fortement rétrécis, celui-ci arrondi et étroit; strie et carène latérales entières; pseudopleures et épipleures assez larges, obliques, strie de l'épipleure effacée au niveau du $2^{\mathrm{e}}$ ou $3^{\mathrm{e}}$ sternite apparent. Ailes complètement atrophiées. Alinotum prolongé en arrière par une longue apophyse médiane (fig. 10). Mésosternum relevé au milieu, son apophyse atteignant le niveau du milieu des cavités coxales II, sa partie médiane limitée de chaque côté par une carène latérale et ornée d'une carène médiane. Mésépimères petits. Mésépisternes bombés, déclinés en avant. Métasternum petit, peu bombé, sa partie médiane étroite (fig. 11). Aires derrière les cavités coxales II pas développées. Hanches II et III rapprochées. Suture interne des métépisternes en gouttière profonde. Premier sternite apparent avec une petite carène médiane rétrécie vers la base; aires postcoxales pas développées. Pattes courtes (fig. 12 à 14), fémurs I et II ne dépassant pas le bord du corps nettement plus longs que leurs tibias respectifs: fémur III légèrement plus long que le tibia.

Ce genre se singularise par le pronotum très grand. Il présente plusieurs caractères des Brachynoposoma: élytres soudés non étranglés à la base, fortement rétrécis vers l'apex; ailes atrophiées; alinotum avec une longue apophyse; superficie des pièces sternales du ptérothorax, du métasternum notamment, relativement petite; pattes courtes et assez robustes. Par contre le corps ovale, la conformation des organes buccaux et des antennes, la présence de la suture du clypéus ainsi que le pronotum proportionnellement plus long avec le lobe basal distinct, permettent de séparer facilement Zinda de Brachynoposoma.

TABLEAU DES ZINDA

1 Pronotum peu rétréci en avant. Ponctuation sur les côtés du métasternum très dense et grosse 


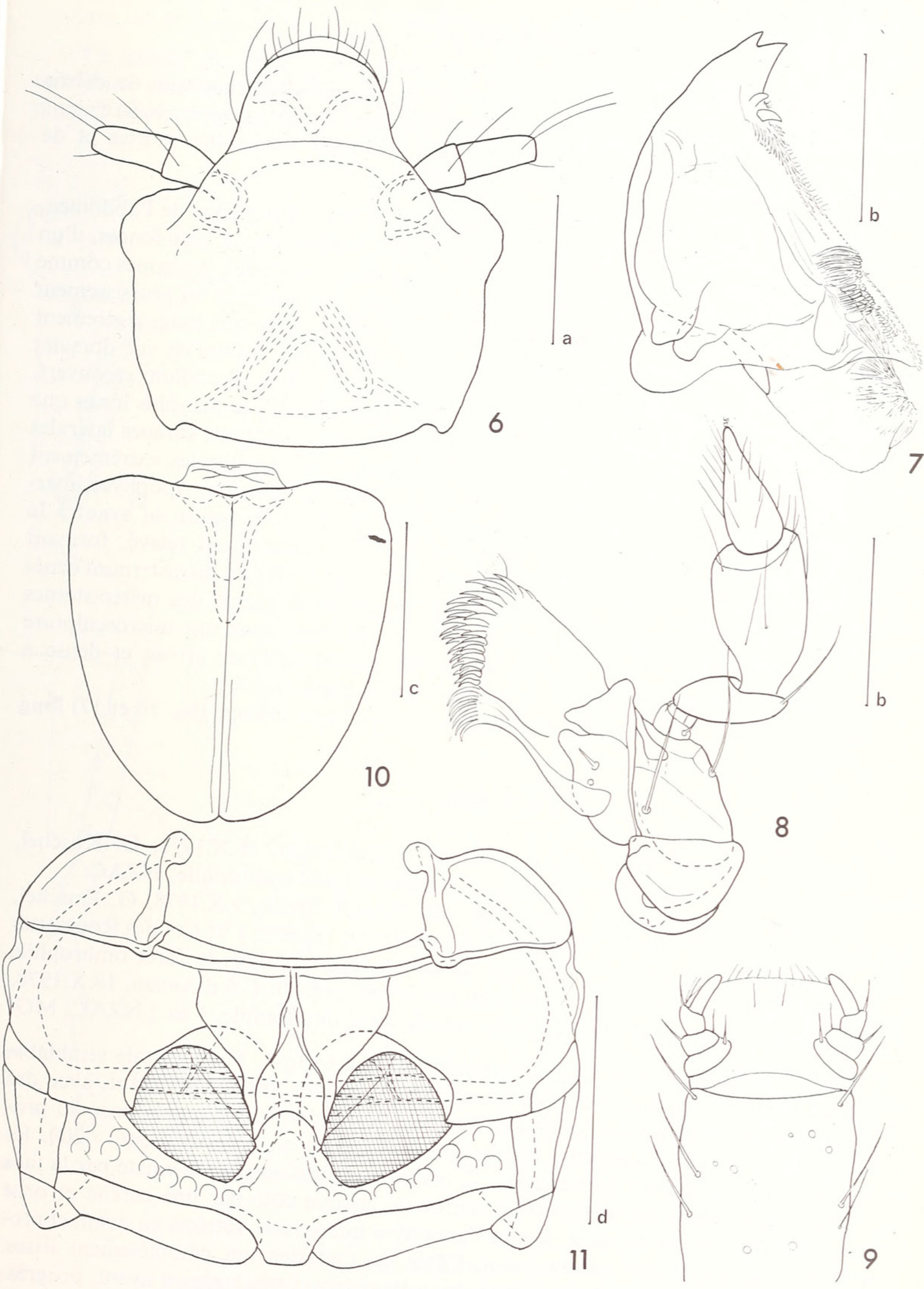

FIg. 6 à 11.

Zinda gen. n. teres sp. n.; 6. tête; 7. mandibule; 8. maxille; 9. labium;
élytres avec alinotum vu par transparence

10. élytres avec alinotum vu par transparence en préparation microscopique; Echelle $\mathrm{a}=0,1$. pièces sternales du ptérothorax. 


\section{Zinda teres sp. $\mathrm{n}$.}

Holotype đ̊: Col d'Amieu 550 m, 15.X.1978, G. Kuschel, tamisage de débris végétaux et de bois pourri dans la forêt ombrophile, ravin avec palmiers; NZAC.

Paratype o: Yahoué, 11.X.1978, G. Kuschel, tamisage de feuilles mortes et de bois pourri avec Syzigium et Sapindaceae, sec; MG.

Longueur 0,85 $\mathrm{mm}$ (tête et abdomen compris, 0,90 mm). Tête, apex de l'abdomen, pattes et articles antennaires I et II ocres. Pronotum et élytres un peu plus foncés, d'un brun rougeâtre clair. Articles antennaires III à XI et palpes jaunâtres. Antennes comme fig. 15. Ponctuation de la face dorsale du corps très fine, peu marquée au grossissement $\times 100$. Pronotum à la base large de $0,55 \mathrm{~mm}$, nettement plus large que long, légèrement rétréci en avant; bord latéral peu convexe; carènes latérales pas visibles en vue dorsale; strie du bord apical bien marquée sur les côtés, effacée au milieu. Scutellum recouvert. Elytres longs sur la suture de $0,50 \mathrm{~mm}$ (en vue dorsale seulement un peu plus longs que le pronotum), à la base presque aussi larges que la base du pronotum; carènes latérales visibles en vue dorsale sur presque toute leur longueur; stries suturales extrêmement fines, courtes, effacées un peu avant le milieu de la longueur suturale. Apophyse intercoxale du mésosternum ornée d'une dépression assez profonde tangente en avant à la carène médiane, en arrière au bord apical. Milieu du métasternum relevé, formant une aire plate, lisse et glabre, légèrement déclinée en arrière. Côtés du métasternum ornés de gros points très serrés. Dépression longitudinale au bord interne des métépisternes assez étroite, de même largeur sur toute sa longueur. Sternites avec une microsculpture formée de points. Ponctuation du premier sternite apparent assez grosse et dense à proximité de la base, progressivement plus fine vers le bord apical.

Mâle. Articles 1 à 3 des tarses antérieurs à peine élargis. Edéage (fig. 16 et 17) long de $0,24 \mathrm{~mm}$.

\section{Zinda abdita sp. $\mathrm{n}$.}

Holotype ô: Table Unio 800 m, près du Col d'Amieu, 16.X.1978, G. Kuschel, tamisage de débris végétaux et de bois pourri dans la forêt ombrophile; NZAC.

Paratypes: comme holotype, 2 đิ 2 ㅇ; Mt. Koghis 700 m, 9.X.1978, G. Kuschel, tamisage de feuilles mortes dans la forêt ombrophile, sur la crête, 1 + ; Col des Roussettes 400 m, 26.X.1978, G. Kuschel, tamisage de débris végétaux dans la forêt ombrophile à proximité d'un cours d'eau, 1 +; Mt. Rembai 700 m, près du Col d'Amieu, 18.X.1978, G. Kuschel, tamisage de débris végétaux dans la forêt ombrophile, 1 ex.; NZAC, MG.

Longueur 0,80-0,90 mm, largeur maximum 0,55-0,64 $\mathrm{mm}$. Face dorsale semblable à celle de teres; le pronotum est cependant nettement plus atténué en avant, avec des côtés régulièrement convexes; les élytres à la base sont aussi larges que la base du pronotum, leur ponctuation presque effacée à peine visible au grossissement $\times 200$, les stries suturales sont encore plus courtes. Z. abdita est toutefois bien distincte par la face ventrale: milieu relevé du métasternum limité de chaque côté par une carène et orné d'une dépression profonde, large devant l'apophyse intercoxale, rétrécie en avant et prolongée sur l'apophyse du mésosternum. Côtés du métasternum complètement lisses. Dépression longitudinale au bord interne des métépisternes très large en avant, progressivement rétrécie en arrière. Premier sternite apparent non ponctué. Edéage (fig. 18 et 19) long de 0,22-0,24 mm, plus grêle que chez teres, son sac interne muni d'un simple flagellum droit. 


\section{Scaphisoma Leach}

Ce genre, particulièrement riche dans les régions équatoriales et tropicales, est représenté en Nouvelle-Calédonie par 27 espèces endémiques, quoi que ailées, dont la plupart vivent dans les débris végétaux en décomposition sur les sols des forêts ombro-
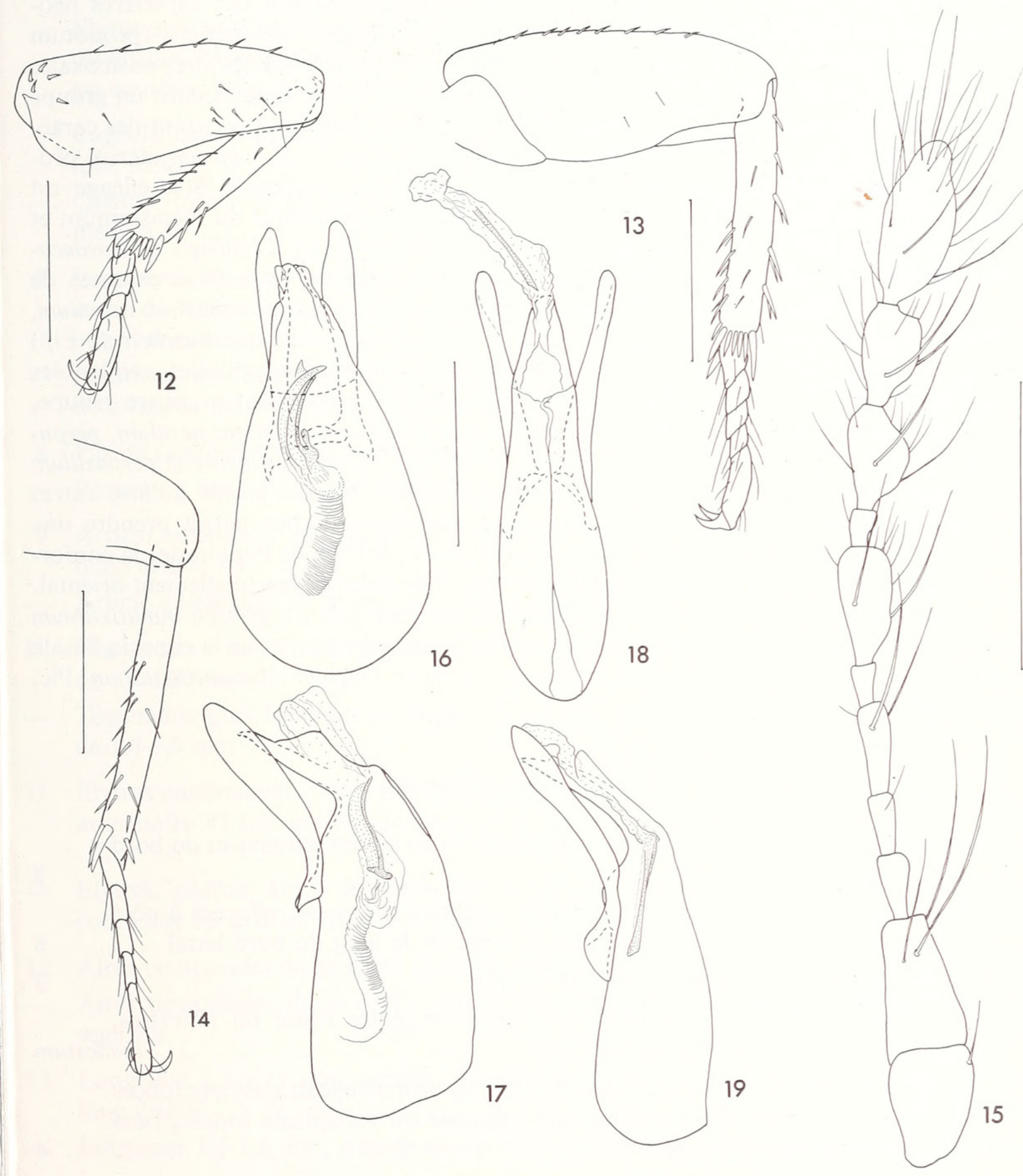

FIG. 12 à 19.

Zinda gen. n.; 12. Z. teres sp. n., patte antérieure, 우

13. Z. teres sp. n., patte intermédiaire, $\circ ; 14$. Z. teres sp. n., patte postérieure,, ;

15. $Z$. teres sp. n., antenne; 16 et 17 . $Z$. teres, sp. n., édéage;

18 et 19. $Z$. abdita sp. n., édéage. Echelle $=0,1 \mathrm{~mm}$. 
philes. Ces espèces présentent un organe copulateur mâle d'un type primitif (sauf chez nanulum et pallens), avec le lobe médian et les paramères peu différenciés par rapport aux espèces connues de Papouasie et d'Australie. Par conséquent, l'étude des édéages (en préparations, indispensables pour l'identification) ne permet guère d'évaluer leur degré d'affinité.

Ainsi 10 espèces, reticulatum, franzi, simulans, consimile, viduum, alternans, filium, variabile, kuschelianum et excellens, très semblables et présentant des caractères néogénétiques inhabituels (apomorphies) au sein des Scaphisoma mélanésiens (pronotum avec microsculpture formée de points, rangée de gros points au bord des aires postcoxales II prolongée sur le bord antérieur du métasternum) doivent être réunies dans un groupe très homogène sans doute monophylétique. Leurs édéages montrent pourtant des caractères distinctifs accusés, au niveau du sac interne en particulier. $S$. rugosum, dérivé probablement de ce groupe, présente les mêmes caractères principaux. Son édéage est similaire à celui de viduum mais l'espèce se singularise par les côtés du métasternum et du premier sternite apparent rugueux (également apomorphie). L'édéage de novaecaledonicum ressemble assez à celui de rugosum, l'ensemble des caractères externes de ces espèces est par contre très différent. Sept espèces (diaphanum, concolor, aemulum, transparens, confrater, modestum et une espèce pas nommée, ainsi que kuscheli de Fiji) de petite taille, au corps peu convexe, avec les stries suturales raccourcies, semblables par la ponctuation et par la conformation des antennes, constituent un autre groupe, affilié peut-être au groupe australien instabile. Les affinités de validum, notulum, perpusillum, funebre, remingtoni et eximium restent incertaines; notulum, funebre et perpusillum paraissent s'approcher de petersoni Löbl de la Nouvelle-Irlande plutôt qu'aux autres espèces sympatriques; eximium avec les palpes maxillaires trapus paraît prendre une place tout à fait isolée. $S$. pallens se rapproche au dissimile Löbl de Papouasie; la conformation de leurs édéages indique l'affinité avec le groupe unicolor essentiellement oriental. $S$. nanulum pourrait se placer par ses caractères externes près du groupe minutissimum mais il présente un édéage bien distinct avec une grande apophyse sur la capsule basale comme chez certaines espèces asiatiques du groupe unicolor (brunneonotatum Pic, maindroni Achard par exemple).

\section{TABLEAU DES SCAPHISOMA}

1 Stries suturales des élytres courtes, effacées bien en arrière du niveau du bord apical du lobe basal du pronotum

- Stries suturales des élytres plus longues, courbées à la base, effacées à côté du lobe basal du pronotum ou prolongées encore le long du bord basal . .

2 Elytres, parfois aussi le pronotum, bicolores . . . . . . . . . . . . . .

- Pronotum et élytres entièrement d'un brun rougeâtre foncé ou noirâtres, avec le bord apical des élytres plus clair . . . . . . . . . . . modestum

3 Corps clair, face dorsale jaunâtre, ocre ou d'un brun rougeâtre, élytres foncés sur la base et ornés chacun d'une tache apicale ou subapicale foncée, base du pronotum parfois foncée

- Téguments du corps d'un brun plus ou moins foncé, élytres avec chacun une grande tache et l'apex clairs

4 Partie distale du lobe médian de l'édéage large, non ou peu déclinée par rapport à la capsule basale; paramères en vue dorsale non ou très légèrement élargis dans leur moitié apicale. 
Partie distale du lobe médian étroite et déclinée; paramères en vue dorsale fortement élargis dans leur moitié apicale

concolor

5 Chaque élytre avec une tache plus petite, située dans la moitié basale du disque. Partie distale du lobe médian très longue et fortement déclinée, son apex en vue latérale brusquement rétréci et sinué, dépassant en arrière les paramères

aemulum

Elytre avec une tache plus grande, étendue nettement en arrière du milieu du disque. Partie distale du lobe médian non ou peu déclinée, à l'apex obtuse ou courbée; paramères dépassant en arrière l'apex du lobe médian

6 Capsule basale large et assez grande, plus longue que la partie distale du lobe médian

- Capsule basale petite, nettement plus courte que la partie distale de lobe médian

transparens

7 Paramères en vue dorsale sinués, élargis au milieu et à l'apex. Sac interne muni de longues épines courbées bien chitinisées et groupées en faisceaux et en rangées .

- Paramères en vue dorsale non sinués, légèrement arqués vers la ligne médiane, de même largeur depuis le rétrécissement basal jusqu'à l'apex . . . . species a

8 Pas de microsculpture sur le pronotum . . . . . . . . . . . . . . . . . . . . 9

— Pronotum orné d'une microsculpture formée de points . . . . . . . . . 17

9 Article antennaire IV très petit, à peu près aussi long ou un peu plus long que l'article III . . . . . . . . . . . . . . . . . . . . . . . . . . . 10

— Article antennaire IV grêle, nettement plus long que l'article III . . . . . 12

10 Téguments très clairs, entièrement d'un ocre jaunâtre. Petite espèce longue de $1,2-1,25 \mathrm{~mm}$. . . . . . . . . . . . . . . . . . . . . . . . . pallens

- Téguments plus foncés, face dorsale bicolore. Espèces plus grandes longues de $1,4-1,9 \mathrm{~mm}$

11 Elytres entièrement d'un brun rougeâtre clair, pronotum plus foncé. Article antennaire VI très grand, plus long que les articles III à V réunis

novaecaledonicum

- Elytres, parfois aussi le pronotum, bicolores. Articles antennaire VI plus court que les articles III à $\mathrm{V}$ réunis . . . . . . . . . . . . . . . . . eximium

12 Aires postcoxales du premier sternite apparent grandes et convexes . . . . 13

- Aires postcoxales du premier sternite étroites, parallèles au bord basal du segment .

13 Longueur 1,1-1,25 mm. Article antennaire VI aussi long ou un peu plus long que V . . . . . . . . . . . . . . . . . . . . . . . . . perpusillum

- Longueur 1,5-1,6 mm. Article antennaire VI nettement plus long que V . funebre

14 Longueur 1,0-1,15 mm. Corps entièrement d'un brun rougeâtre clair. Aires derrière les cavités coxales II étroites, longues de $0,02 \mathrm{~mm}$. . . . . . . nanulum

- Longueur 1,45-2,25 mm. Téguments du corps, ou de l'avant-corps au moins, plus foncés à noirs; élytres souvent ornés de taches. Aires derrière les cavités coxales II plus grandes et convexes, longues de 0,04-0,07 mm 
15 Longueur 2,1-2,25 mm. Elytres noirâtres, chacun avec une tache posthumérale et une bande transversale subapicale jaunâtre validum

- Longueur 1,45-1,9 mm. Elytres ocres ou d'un brun rougeâtre à noirâtre, unicolores ou chacun avec une ou deux petites taches foncées subapicales

16 Elytres aussi foncés que le pronotum, parfois avec une ou deux petites taches subapicales claires

notulum

- Elytres nettement plus clairs que le pronotum, ocres ou d'un brun rougeâtre, soit unicolores ou plus foncés sur la base, sur sa partie interne notamment, et souvent ornés d'une petite tache subapicale foncée

remingtoni

17 Côtés du métasternum et du premier sternite apparent avec une ponctuation très fine

- Côtés du métasternum et du premier sternite rugueux

rugosum

18 Ponctuation du pronotum nettement plus dense et grosse près du bord apical qu'au milieu du disque

- Ponctuation du pronotum très fine et régulièrement espacée sur toute la surface

19 Sac interne de l'édéage muni d'une pièce chitinisée grêle bifurquée. Apex des paramères en vue dorsale brusquement courbé vers la ligne médiane . viduum

- Sac interne de l'édéage avec une pièce chitinisée robuste et bilobée. Paramères légèrement arqués à l'apex variabile

20 Paramères de l'édéage grêles, bien chitinisée

- Face interne des paramères fortement élargie et membraneuse excellens

21 Face ventrale du lobe médian ornée de quelques denticules bien marqués en vue latérale

alternans

- Face ventrale du lobe médian non denticulée .

22 Capsule basale de l'édéage avec deux grands tubercules ventraux saillants en arrière, situés au-dessus de la base des paramères reticulatum

- Pas de tubercules saillants en arrière sur la capsule basale de l'édéage

23 Paramères de l'édéage en vue dorsale à peu près de même largeur sur leur moitié apicale, parfois de même largeur sur presque toute leur longueur . .

- Paramères progressivement rétrécis vers l'apex

24 Sac interne membraneux et simple. Lobe médian grêle consimile

- Sac interne de l'édéage épineux, denticulé ou pourvu d'une pièce chitinisée asymétrique. Lobe médian plus ou moins large

filium

25 Face ventrale du lobe médian non échancrée

- Face ventrale du lobe médian profondément échancrée juste en arrière du niveau de la base des paramères (vue latérale) kuschelianum

26 Lobe médian de l'édéage très grand. Sac interne épineux simulans

- Lobe médian plus étroit. Sac interne avec une pièce chitinisée, dépourvu d'épines. 


\section{Scaphisoma diaphanum Löbl}

Scaphisoma diaphanum Löbl, 1973: 313, fig. 7, 8; holotype ơ: Mt. Koghis; Franz.

Matériel étudié (5 ex.): le type unique du Mt. Koghis; Mt. Koghis et Col des Roussettes (Löbl, 1977b); Mt. Koghis 550 m, 7.X.1978, G. Kuschel, tamisage de feuilles mortes et de bois pourri sur un terrain plat, 1 ex.

Longueur 1,2-1,4 $\mathrm{mm}$. Tête et pronotum entièrement d'un brun rougeâtre assez clair à ocre, ou le pronotum plus foncé à noirâtre sur sur la base. Couleur dominante des élytres jaunâtre ou ocre, leur base plus foncée à noirâtre, juste devant l'apex une tache plus ou moins grande assez irrégulière. L'aire basale foncée plus ou moins prolongée le long de la suture en triangle atténué apicalement. Article antennaire IV grêle, 1,8 à 2 fois plus long que III; V nettement plus long et large que IV, VI un peu plus grand que V. Pas de microsculpture sur le pronotum. Elytres avec les stries suturales profondes, courtes, brusquement effacées en arrière du quart basal de la longueur suturale; carènes latérales distinctes sur toute leur longueur en vue dorsale; ponctuation discale assez éparse et très fine, semblable à celle du pronotum sur la moitié basale, progressivement plus dense et grosse du milieu vers le tiers ou le quart apical, sur le quart apical bien distincte et très dense. Aires derrière les cavités coxales II longues de $0,05 \mathrm{~mm}$, non ponctuées sur leur bord. Aires postcoxales du premier sternite apparent longues de 0,07-0,09 mm, convexes. Edéage long de 0,40 mm, assez plat. Lobe médian presque droit, avec la partie distale large, l'apex obtus. Sac interne simple, membraneux, dépourvu de denticules ou de pièces chitinisées. Paramères assez grêles, en vue dorsale légèrement sinués, dépassant nettement l'apex du lobe médian, leur base prolongée proximalement.

\section{Scaphisoma concolor sp. $\mathrm{n}$.}

Holotype ô: Table Unio $800 \mathrm{~m}$, près du Col d'Amieu, 18.X.1978, G. Kuschel, tamisage de débris végétaux et de bois pourri; NZAC.

Paratypes: comme holotype, 1 ô 4 क; Mt. Koghis 500 m, 7.X.1978, G. Kuschel, sur un tronc pourri, 7 ô 8 of Mt. Koghis 650 m, 12.X.1978, J. S. Dugdale, sur un tronc avec champignons, 2 o 1 \%; Mt. Rembai 800 m, 21.X.1978, G. Kuschel, tamisage de bois pourri sur un chemin, 2 ơ; NZAC, MG; Ile des Pins, 23-27.II.1977, J. Balogh, 1 ô; TMB.

Longueur 1,15-1,3 mm. Corps peu convexe. Téguments clairs et assez transparents. Tête et pronotum entièrement d'un ocre clair. Elytres ocres ou jaunâtres, ornés chacun d'une tache basale brune plus ou moins foncée et d'une assez petite tache foncée subapicale ou apicale. Tache basale triangulaire, effacée sur les côtés du disque, plus ou moins prolongée le long de la suture, mais ne dépassant pas le quart basal de la longueur suturale. Antennes et pattes très claires. Antennes de longueur assez variable, rapport des longueurs des articles: III 4-5 : IV 6-8 : V 9-13 : VI 14-18 : VII 18-20 : VIII 12-15 : IX 18-21 : X 18-21 : XI 30-33; IV relativement large, à peu près deux fois ou un peu moins que 2 fois plus long que large; $\mathrm{V}$ nettement plus large que IV, 2,5-3 fois plus long que large; VI encore bien plus large que V, 3 fois plus long que large; VII 2,5-3 fois plus long que large; VIII à peu près 2,5 plus long que large, très légèrement plus large que VI; XI bien 4 fois à 4,5 fois plus long que large, à peu près aussi large que VII. Pronotum à la base de $0,75-0,81 \mathrm{~mm}$; côtés régulièrement arrondis; carènes latérales non visibles en vue dorsale; pas de microsculpture; ponctuation assez éparse et très 
fine, à peine visible au grossissement $\times 50$. Extrémité du scutellum libre. Elytres longs sur la suture de 0,80-0,90 $\mathrm{mm}$ (longueur maximum 0,89-0,95 $\mathrm{mm}$ ); largeur maximum $(0,89-0,98 \mathrm{~mm})$ située juste derrière le tiers basal; bord latéral convexe entre la base et le tiers médian, ensuite rectiligne ou très légèrement arrondi; carènes latérales bien visibles sur toute leur longueur en vue dorsale; bord apical légèrement arrondi; angles apicaux internes situés au même niveau que les angles externes; bord sutural non ou à peine relevé; stries suturales assez profondes, ponctuées, courtes, effacées à 0,05-0,15 mm derrière le scutellum; espace entre la suture et les stries suturales un peu bombé; ponctuation discale sur le tiers basal à peu près aussi fine que celle du pronotum (sauf sur la tache basale où elle est nettement plus distincte), ensuite progressivement plus dense et grosse, sur la tache subapicale très dense. Pygidium orné d'une microsculpture formée de points; ponctuation très fine. Mésépimères plus longs que la distance entre eux et les hanches II. Métasternum nettement bombé au milieu, avec deux dépressions médioapicales très superficielles; ponctuation partout très fine et assez éparse. Aires derrière les cavités coxales II longues de 0,04-0,05 mm, non ponctuées sur leur bord. Métépisternes plats, larges de $0,10-0,12 \mathrm{~mm}$ en arrière, rétrécis en avant, leur suture interne très légèrement concave ou presque droite. Premier sternite apparent sans microsculpture; ponctuation sur ses côtés comme celle du métasternum, sur sa partie médiane un peu plus distincte et très dense; aires postcoxales longues de 0,07-0,08 mm, arrondies, avec des points marginaux fins à très fins. Sternites suivants ornés d'une microsculpture formée de points. Tibias droits et grêles.

Mâle. Articles 1 à 3 des tarses antérieurs un peu élargis. Edéage (fig. 20 et 21) long de 0,37-0,47 $\mathrm{mm}$, peu chitinisé.

Cette espèce est très semblable à diaphanum; elle s'en distingue par les stries suturales des élytres moins raccourcies, le pronotum toujours entièrement ocre et par la conformation de l'édéage notamment.

\section{Scaphisoma aemulum Löbl}

Scaphisoma aemulum Löbl, 1973: 312, fig. 5, 6; holotype ô: Table Unio; Franz.

Matériel étudié (26 ex.): type unique de Table Unio; Table Unio $800 \mathrm{~m}$, près du Col d'Amieu, 16.X.1978, G. Kuschel, tamisage de débris végétaux et de bois pourri dans la forêt ombrophile, 1 ex.; Mt. Koghis 550 m, 5.X.1978, G. Kuschel, tamisage de débris végétaux et de bois pourri dans la forêt ombrophile dans un ravin peu profond, 2 ex. et tamisage de débris végétaux dans la zone entre la forêt et le maquis, 1 ex.; Mt. Koghis 500 m, 7.X.1978, G. Kuschel, sur un tronc pourri, 10 ex.; Mt. Koghis, 500-550 m, 9.X.1978, J. S. Dugdale, fauchage dans la forêt, 2 ex.; Mt Koghis 550 m, 5.X.1978, J. S. Dugdale, sur un champignon, 1 ex.; Mt. Koghis 650 m, 12.X.1978, J. S. Dugdale, sur un tronc avec champignons, 7 ex.; Mt. Koghis, 12.X.1978, J. C. Watt, au-dessous d'un tronc, 1 ex.

Longueur 0,95-1,15 mm. Corps d'un brun assez foncé ou très foncé; pronotum parfois légèrement plus clair que la tête ou la base des élytres. Elytres ornés chacun d'une grande tache ocre ou jaunâtre plus ou moins transparente, mal limitée et située entièrement dans la moitié basale du disque ou dépassant un peu seulement le milieu. Apex des élytres assez clair, d'un brun rougeâtre ou jaunâtre. Abdomen d'un brun plus ou moins foncé avec les derniers segments jaunâtres, ou entièrement clair. Article antennaire IV petit, à peu près 2 fois plus long que large et généralement 1,5 plus long que III; V de même longueur que III et IV réunis, nettement plus large que IV, 2,5 fois plus long que large; VI nettement plus long mais à peine plus large que $\mathrm{V}$, à peu près 3 fois plus long 
que large; VIII un peu plus court mais aussi large que VI, 2,5-3 fois plus long que large; XI long, 4-4,5 plus long que large. Pas de microsculpture sur le pronotum. Carènes latérales du pronotum généralement non visibles en vue dorsale, parfois distinctes au milieu; celles des élytres bien marquées sur toute leur longueur. Elytres avec les stries suturales courtes, effacées à $0,14-0,18 \mathrm{~mm}$ derrière le niveau du scutellum; ponctuation discale très fine, semblable à celle du pronotum sur la moitié basale, progressivement plus distincte du milieu vers l'apex, encore bien fine sur le tiers apical. Aires derrière les cavités coxales II longues de 0,04-0,05 mm. Aires postcoxales du premier sternite apparent très convexes, longues de 0,06-0,08 mm. Edéage long de 0,41-0,52 $\mathrm{mm}$. Lobe médian avec la capsule basale assez grande, la partie distale très longue, brusquement déclinée à sa base, rétrécie et sinuée (vue latérale) à l'apex. Sac interne muni d'un long flagellum, ses membranes ornées de denticules. Paramères très grèles, en vue dorsale presque droits, sinués en vue latérale, ne dépassant pas l'apex du lobe médian.

\section{Scaphisoma transparens Löbl}

Scaphisoma transparens Löbl, 1973: 310; fig. 3, 4; Holotype ơ: Table Unio; Franz.

Matériel étudié (21 ex): types de Table Unio et du Mt. Panié; Ile des Pins, 23-27.II. 1977, J. Balogh, 1 ex.; Table Unio 800 m, près du Col d'Amieu, 16.X.1978, G. Kuschel, tamisage de débris végétaux et de bois pourri dans la forêt ombrophile, 12 ex.; Mt. Koghis, 500 m, 7.X.1978, G. Kuschel, sur un tronc pourri, 2 ex.; Mt. Koghis, 12.X.1978, J. C. Watt, au-dessous d'un tronc, 1 ex.

Cette espèce présente les mêmes caractères ectosquelettiques que aemulum; en diffère par les élytres ornés chacun d'une tache plus grande, dépassant nettement le milieu du disque, atteignant parfois le tiers apical, et par la ponctuation plus grosse sur le tiers apical. Edéage pourtant bien distinct, plus petit, long de 0,25-0,30 mm le plus souvent mais long de 0,34-0,38 mm chez quatre exemplaires. Lobe médian grêle, avec petite capsule basale et longue partie distale à peine déclinée, arrondie à l'apex. Sac interne muni d'un flagellum peu chitinisé visible en érection seulement; membanes avec des denticules extrêmement fins et de nombreux spicules à l'apex. Paramères grêles, en vue dorsale sinués, élargis un peu devant le niveau de l'apex du lobe médian, ensuite assez fortement rétrécis, dépassant nettement en arrière le lobe médian.

Les caractères généraux ne permettent pas de distinguer transparens des deux espèces suivantes; par conséquent je n'ai pas pu identifier 26 femelles recueillies au Table Unio et au Mt. Koghis, appartenant probablement à ces espèces et celles-ci ne sont pas incluses dans le matériel étudié.

\section{Scaphisoma confrater sp. n.}

Holotype ô: Table Unio 800 m, près du Col d'Amieu, 16.X.1978, G. Kuschel, tamisage de débris végétaux et de bois pourri dans la forêt ombrophile; NZAC.

Paratypes: comme holotype, 2 đ̃; Pic d'Amoa près de Poindimié, 450 m, 26.X.1978, G. Kuschel, tamisage de débris végétaux dans la forêt ombrophile, 1 đo: Mt. Koghis, 12.X.1978, J. C. Watt, au-dessous d'un tronc, 1 §ิ; NZAC et MG.

Longueur 1,0-1,15 mm. Coloration et caractères généraux comme chez transparens, les taches sur les élytres parfois élargies vers l'intérieur et tangentes à la suture. Antennes de longueur assez variable; rapport des longueurs des articles chez l'holotype: III 4 : IV 7 : V 11: VI 13 : VII 17 : VIII 12 : IX $18:$ X $17:$ XI 28. Pronotum à la base large 

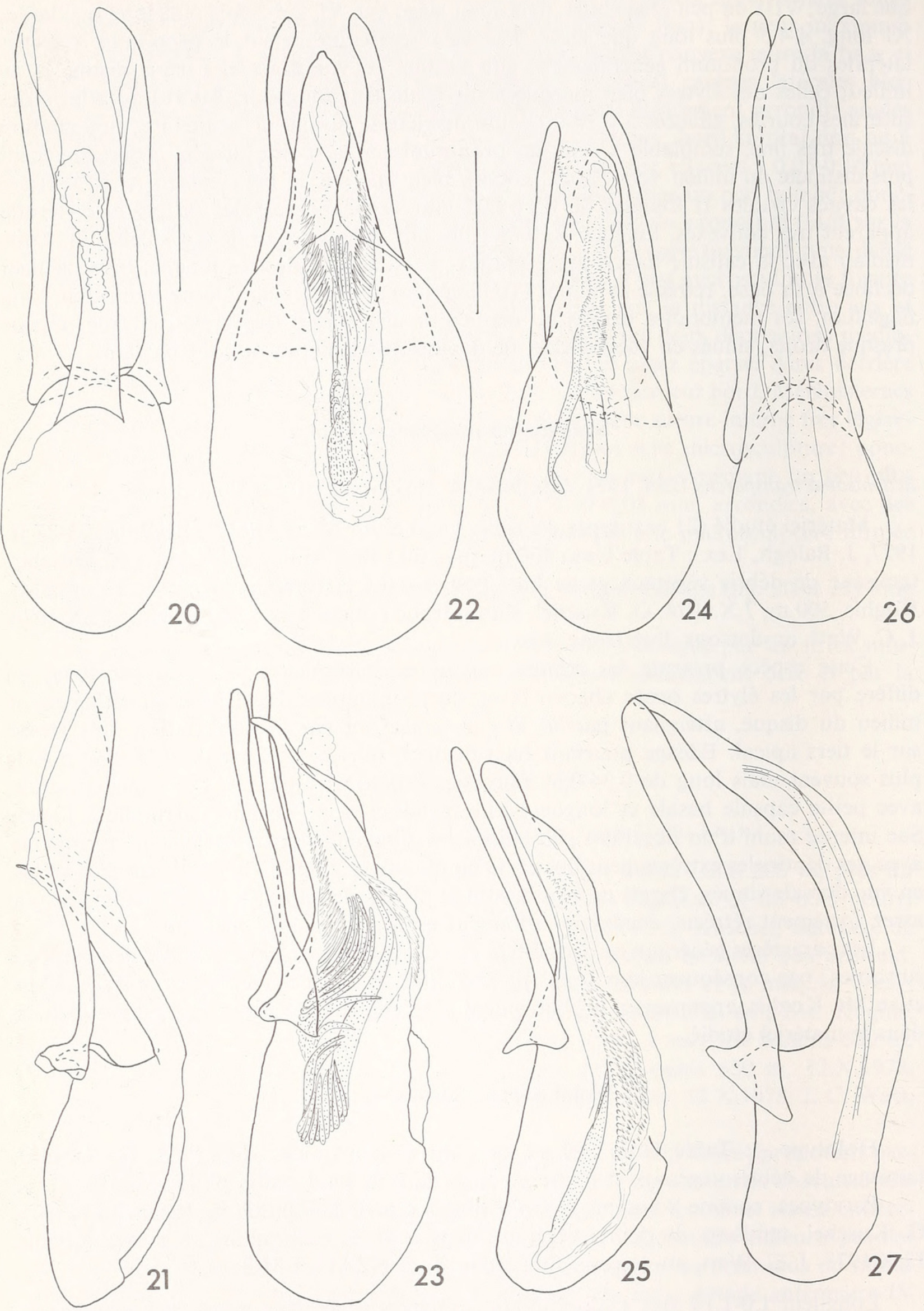

FIG. 20 à 27.

Edéages chez Scaphisoma; 20 et 21. S. concolor sp. n.; 22 et 23. S. confrater sp. n.; 24 et 25 . S. modestum sp. n.; 26 et 27. S. S. notulum Fauvel. Echelle $=0,1 \mathrm{~mm}$. 
de 0,56-0,69 mm, élytres à la suture longs de 0,61-0,75 mm (longueur maximum 0,70$0,82 \mathrm{~mm}$ ), réunis larges de $0,66-0,80 \mathrm{~mm}$. Stries suturales des élytres effacées à $0,12-$ $0,15 \mathrm{~mm}$ derrière le niveau du scutellum. Edéage (fig. 22 et 23) long de 0,35-0,40 mm. Lobe médian avec une capsule basale relativement grande, la partie distale peu déclinée, brusquement courbée à l'apex. Sac interne pourvu d'une longue pièce chitinisée située dorsalement, couverte par des rangées de denticules très fins, et avec deux faisceaux de longues épines courbées, dont un basal, formé d'épines plus robustes, le deuxième situé dans la partie centrale et formé d'épines grêles; deux rangées latérales d'épines progressivement plus fines et courtes prennent naissance au milieu du sac et se prolongent vers la partie distale membraneuse. Paramères sinués, en vue dorsale élargis au milieu et à l'apex, dépassant le lobe médian.

\section{Scaphisoma species $a$.}

Matériel étudié: Mt. Koghis, 12.X.1978, J. C. Watt, au-dessous d'un tronc, 1 đ๋; Col d'Amieu 550 m, 15.X.1978, G. Kuschel, tamisage de débris végétaux et de bois pourri sur une crête, 1 ô.

Ces exemplaires présentent les mêmes caractères généraux que transparens et confrater. Leur édéage, avec le lobe médián semblable à celui de confrater, diffère par les paramères non sinués, en vue dorsale légèrement courbés vers la ligne médiane, de même largeur, de l'apex jusqu'à l'élargissement basal. Le sac interne, étant en érection et donc déformé chez les deux mâles connus, semble être pourvu de deux longues pièces latérales peu chitinisées; ces membranes sont couvertes de très nombreux spicules fins et droits. Il est bien possible que ces exemplaires représentent une espèce pas encore nommée mais il faudrait un matériel en meilleure condition pour la décrire.

\section{Scaphisoma modestum sp. n.}

Holotype §̊: Mt. Rembai 800 m, 21.X.1978, G. Kuschel, tamisage de feuilles mortes et de bois pourri dans la forêt ombrophile; NZAC.

Paratypes: comme holotype, 1 ô et 1 क; Mt. Koghis 650 m, 12.X.1978, J. S. Dugdale, sur un tronc avec champignons, 1 우 Col d'Amieu 550 m, 15.X.1978, G. Kuschel, tamisage de débris végétaux et de bois pourri dans un ravin avec des palmiers dans la forêt ombrophile, $3 \hat{\jmath}, 7$; ; NAC, MG.

Longueur 1,0-1,2 mm. Corps peu convexe, entièrement d'un brun rougeâtre foncé à noirâtre, mais avec cependant le bord apical des élytres et l'apex de l'abdomen plus clairs. Antennes et pattes claires. Rapport longueur des articles antennaires: III 4 : IV 6 : V 10: VI 12: VII 17: VIII 12: IX 18: X 18: XI 25 (holotype); article IV très petit, 2 fois plus long que large; $\mathrm{V}$ un peu plus large que IV, à peu près 3 fois plus long que large; VI nettement plus large que $\mathrm{V}$, pas tout à fait 3 fois plus long que large; VII et VIII chacun trois fois plus long que large, VIII légèrement plus grêle ou aussi large que VI; XI 4 fois plus long que large. Pronotum sans microsculpture, à la base large de 0,63$0,72 \mathrm{~mm}$; côtés légèrement arrondis; carènes latérales en vue dorsale généralement non visibles; ponctuation assez éparse et très fine, à peine marquée au grossissement $\times 50$. Extrémité du scutellum visible. Elytres longs sur la suture de 0,66-0,75 mm (longueur maximum 0,73-0,84); largeur maximum $(0,72-0,86 \mathrm{~mm})$ située derrière le tiers basal; bord latéral convexe dans la moitié basale, rectiligne entre le milieu et l'apex; 
carènes latérales bien visibles en vue dorsale sur toute leur longueur; bord apical légèrement arrondi; angles apicaux internes situés au même niveau ou un peu derrière le niveau des angles externes; bord sutural non relevé; espace entre la suture et la strie suturale plat, orné d'une rangée de points très fins; stries suturales peu profondes, courtes, effacées à $0,14-0,20 \mathrm{~mm}$ derrière le niveau du scutellum; ponctuation discale éparse et très fine dans la partie basale, nettement plus dense et distincte dans le tiers médian, relativement grosse près de l'apex. Ponctuation du pygidium très fine. Mésépimères de même longueur que la distance entre eux et les hanches II. Métasternum sans microsculpture, orné d'une ponctuation dense et très fine, bombé au milieu. Aires derrière les cavités coxales II longues de 0,05-0,06 mm, dépourvues de points marginaux. Métépisternes plats, larges en arrière de 0,08-0,10 $\mathrm{mm}$, nettement rétrécis en avant; suture interne droite. Partie médiane du premier sternite apparent orné d'une microsculpture formée de points; pas de microsculpture sur ses côtés; aires postcoxales longues de 0,08-0,10 mm, arrondies, les points marginaux fins. Tibias grêles et droits.

Mâle. Articles 1 à 3 des tarses antérieurs légèrement élargis. Edéage (fig. 24 et 25) long de 0,35-0,40 mm.

Cette espèce rappelle à première vue kuscheli Löbl de Fiji, mais en diffère par l'article antennaire IV nettement plus court, les élytres à carènes latérales bien marquées en vue dorsale, ornés d'une ponctuation discale plus grosse et dense, les aires postcoxales plus grandes, le premier sternite apparent pourvu d'une microsculpture. Les édéages de ces deux espèces sont différents, notamment par la pièce chitinisée du sac interne et les paramères grêles de l'espèce nouvelle, tandis que chez kuscheli le sac interne est inerme et les paramères sont élargis vers l'apex.

\section{Scaphisoma validum Löbl}

Scaphisoma validum Löbl, 1973: 317, fig. 13, 14; holotype đ̊: Roche d'Ouaième; Franz.

Matériel étudié ( 2 ex.): les types trouvés à Roche d'Ouaième près d'Hienghène.

Longueur 2,1-2,25 mm. Noirâtre, élytres ornés chacun d'une tache posthumérale et d'une bande transversale apicale jaunâtres bien limitées, cette dernière bilobée en avant. Article antennaire IV grêle, à peu près 2 fois plus long que III, V un peu plus large que IV et légèrement plus long que III et IV réunis, VI plus court et très légèrement plus large que V. Pas de microsculpture sur le pronotum. Elytres avec une ponctuation discale fine sur leur tiers basal, progressivement plus grosse vers l'apex, grosse et très dense sur le tiers apical; stries suturales courbées à la base mais pas prolongées le long du bord basal. Aires derrière les cavités coxales II longues de 0,05-0,06 mm, celles du premier sternite apparent extrêmement étroites, à peine longues de 0,02 $\mathrm{mm}$. Edéage long de $0,62 \mathrm{~mm}$. Lobe médian très grêle, capsule basale petite, partie distale longue, légèrement courbée, atténuée en pointe à l'apex. Sac interne simple, membraneux. Paramères grêles et presque droits.

\section{Scaphisoma perpusillum Löbl}

Scaphisoma perpusillum Löbl, 1973: 316, fig. 11, 12; holotype đ̊: Tiouandé; Franz.

Matériel étudié (37 ex.): les types de Tiouandé; Col des Roussettes (LöBL 1977b); 7 miles au sud-est de La Foa (LöвL 1974); Mt. Koghis 550 m, 7.X.1978, G. Kuschel, tamisage de feuilles mortes et de bois pourri sur un terrain plat, 1 ex.; Mt. Koghis 
650 m, 12.X.1978, J. S. Dugdale, sur un tronc avec champignons, 9 ex.; Mt. Koghis 500 m, 7.X.1978, J. C. Watt, sur un tronc, 1 ex.; Mt. Koghis, 12.X.1978, J. C. Watt, au-dessous d'un tronc, 1 ex.; Table Unio 800 m, près du Col d'Amieu, 16.X.1978, G. Kuschel, tamisage de débris végétaux dans la forêt ombrophile, 8 ex.; Yahoué, 11.X.1978, G. Kuschel, tamisage de feuilles mortes et de bois pourri avec Syzygium et Sapindaceae, sec, 2 ex.; Mt. Rembai 800 m, 21.X.1978, G. Kuschel, tamisage de bois pourri, 5 ex.

Longueur 1,1-1,25 mm. Corps d'un brun rougeâtre très foncé à noirâtre, apex des élytres d'un brun clair ou jaunâtre sur une zone large de 0,06 à 0,10 mm. Article antennaire IV très grêle, $\mathrm{V}$ à peu près de même longueur mais un peu plus large; VI aussi long ou légèrement plus long et plus large que $\mathrm{V}$. Pronotum sans microsculpture; ponctuation très fine et assez dense. Elytres avec la ponctuation discale plus distincte que celle du pronotum, assez grosse devant l'apex; stries suturales courbées à la base et terminées près du lobe basal du pronotum ou encore légèrement prolongées le long du bord basal. Aires derrière les cavités coxales II bien développées, longues de 0,04-0,05 mm, avec quelques points marginaux très fins. Métasternum orné d'une grosse ponctuation sur sa partie médioapicale. Aires coxales du premier sternite apparent longues de 0,070,08 mm, convexes, avec de gros points marginaux. Edéage long de 0,21-0,25 mm. Lobe médian avec une petite capsule basale prolongée par une saillie ventro-apicale et une longue partie apicale régulièrement courbée, obtus au sommet. Sac interne simple, muni d'un flagellum peu chitinisé. Paramères grêles, pas ou dépassant de peu l'apex du lobe médian, presque droits, leur base prolongée proximalement.

\section{Scaphisoma notulum Fauvel}

Scaphosoma notula Fauvel, 1903: 292; holotype +: Nouméa; IRSN. Scaphisoma notulum; LöBL 1969: 2, fig. 3.

Matériel étudié (5 ex.): le type unique de Nouméa; Mt. Koghis 700 m, crête, 9.X.1978 G. Kuschel, tamisage de feuilles mortes dans la forêt ombrophile, 1 ex. et tamisage de feuilles mortes sur une pente raide, 1 ex.; Col de Mouirange, 11.X.1978, G. Kuschel, tamisage de feuilles mortes et de bois pourri dans un ravin avec des brousailles, 2 ex.

Espèce très variable. Longueur 1,45-1,9 mm. Corps d'un brun rougeâtre plus ou moins foncé à noirâtre. Elytres comme le pronotum et la tête mais avec l'apex plus clair, ou chacun encore avec une ou deux petites taches subapicales claires. Article antennaire IV grêle, à peu près 2 fois plus long que III, V un peu plus long et plus large que IV, nettement plus court et moins large que VI. Pas de microsculpture sur le pronotum. Elytres avec les stries suturales courbées à la base et plus ou moins prolongées le long du bord basal, ne dépsasant pas à l'extérieur de la moitié interne du disque, parfois terminée à côté du lobe du pronotum; ponctuation fine à très fine, nettement plus grosse que celle du pronotum sauf sur le quart ou le tiers apical où elle est assez grosse. Ponctuation du métasternum et des sternites très fine et éparse. Pas de dépressions médio-apicales sur le métasternum. Aires derrière les cavités coxales II longues de 0,04-0,05 mm, les points marginaux assez gros. Métépisternes larges de 0,10-0,15 mm en arrière, atténués en avant, leur suture interne légèrement convexe. Premier sternite apparent sans microsculpture, ses aires postcoxales étroites, longues de 0,03-0,04 mm. Les sternites suivants et le pygidium ornés d'une microsculpture formée de points. Edéage (fig. 26 et 27) long de 0,37-0,42 mm. 


\section{Scaphisoma funebre sp. $\mathrm{n}$.}

Holotype đ̃: Mt. Koghis 550 m, 5.X.1978, G. Kuschel, tamisage de débris végétaux et de bois pourri dans la forêt ombrophile; NZAC.

Paratypes: comme holotype, 1 ô, 1 \%; NZAC, MG.

Longueur 1,5-1,6 mm. Corps assez convexe, noirâtre, le bord apical des élytres et l'apex de l'abdomen plus clairs. Fémurs et tibias d'un brun rougeâtre foncé, tarses et articles antennaires I à IV d'un ocre clair, articles V à XI d'un brun assez clair. Rapport des longueurs des articles antennaires: III 6 : IV 12 : V 15 : VI 16 : VII 21 : VIII 15 : IX 20 : X 20 : XI 28 (holotype); article IV grêle, 4 fois plus long que large; $\mathrm{V}$ nettement plus large que IV, presque 4 fois plus long que large; VI très légèrement plus large que $\mathrm{V}$, à peu près 3,5 fois plus long que large; VII à peine 2,5 fois plus long que large; VIII aussi large que VI, à peu près 3,5 fois plus long que large; XI 3 fois plus long que large. Pronotum à la base large de 0,90-0,94 mm, assez rétréci en avant; côtés régulièrement arrondis; carènes latérales en vue dorsale non visibles; pas de microsculpture; ponctuation dense et très fine à peine marquée au grossissement $\times 50$. Scutellum couvert par le lobe du pronotum. Elytres à la suture longs de 0,96-1,02 mm (longueur maximum 1,07-1,13 mm); largeur maximum (1,02-1,10 mm) situés juste derrière le quart basal, assez fortement rétrécis vers l'apex; côtés légèrement arrondis; carènes latérales en vue dorsale non visible; bord apical arrondi; angles apicaux internes situés au même niveau que les angles externes; espace entre la suture et les stries suturales relevé, sauf en avant où il est plat, orné d'une rangée de points fins; stries suturales assez profondes, courbées à la base et terminées près du lobe basal du pronotum; ponctuation discale dense et fine, sur la base très fine. Pygidium pourvu d'une microsculpture formée de points; sa ponctuation éparse, assez fine dans la moitié basale très fine dans la moitié apicale. Mésépimères un peu plus courts que la distance entre eux et les hanches II. Métasternum très légèrement bombé au milieu, avec deux dépressions médio-apicales; ponctuation éparse et très fine sur les côtés et sur le milieu, nettement plus grosse et dense entre les hanches III. Aires derrière les cavités coxales II longues de $0,06 \mathrm{~mm}$, avec des points marginaux très fins. Métépisternes à peine bombés, rétrécis en avant, larges en arrière de 0,11-0,13 mm, leur suture interne légèrement convexe. Premier sternite apparent avec une ponctuation très fine et éparse sur les côtés, plus grosse et dense au milieu, dépourvu de microsculpture; aires postcoxales longues de 0,12 mm, arrondies avec de gros points marginaux. Sternites suivants ornés d'une microsculpture formée de points bien marqués. Tibias droits, assez grêles.

Mâle. Articles 1 à 3 des tarses antérieurs à peine élargis. Edéage (fig. 28 et 29) long de $0,28-0,29 \mathrm{~mm}$.

Cette nouvelle espèce prend place semble-t-il près de perpusillum; elle en diffère principalement par la taille plus grande, par la ponctuation des élytres plus grosse et par les aires postcoxales du premier sternite apparent plus longues.

\section{Scaphisoma remingtoni Löbl}

Scaphisoma remingtoni Löbl, 1974: 405, fig. 1, 2; holotype $\delta$ : 7 miles au sud-est de La Foa; FMNH.

Matériel étudié (52 ex.): les types; La Crouen et Mt. Koghis (LöBL 1977b); Mt. Koghis 550 m, 5.X.1978, G. Kuschel, tamisage de débris végétaux et de bois pourri dans la forêt ombrophile, 6 ex.; même donnée, mais tamisage de débris végétaux dans 
la zone entre la forêt et le maquis, 1 ex.; Mt. Koghis 500 m, 7.X.1978, G. Kuschel, sur un tronc pourri, 7 ex.; Mt. Koghis 550 m, 7.X.1978, G. Kuschel, tamisage de feuilles mortes et de bois pourri sur un terrain plat, 1 ex.; Mt. Koghis, 500-550 m, 9.X.1978, J. S. Dugdale, fauchage dans la forêt, 1 ex.; Mt. Koghis, 650 m, 12.X.1978, J. S. Dugdale sur un tronc avec champignons, 1 ex.; Table Unio 800 m, près de Col d'Amieu, 16.X.1978, G. Kuschel, tamisage de débris végétaux et de feuilles mortes dans la forêt ombrophile, 2 ex.; Mt. Rembai 800 m, 21.X.1978, G. Kuschel, tamisage de bois pourri sur le chemin, 20 ex.

Longueur 1,6-1,9 mm. Tête et pronotum d'un brun noirâtre ou noirs. Elytres ocres ou d'un brun rougeâtre, parfois unicolores, généralement ornés chacun d'une petite tache foncée subapicale et d'une plus grande tache basale noirâtre, celle-ci plus ou moins large sur la base, tangente à la suture et prolongée en triangle atténué en arrière. Articles antennaires IV et $\mathrm{V}$ très grêles et de même longueur ou $\mathrm{V}$ un peu plus long que IV, VI un peu plus grand que V. Pas de microsculpture sur le pronotum. Elytres avec les stries suturales prolongées le long de la base vers le quart externe de la largeur basale; ponctuation discale fine mais nettement plus distincte que celle du pronotum. Aires derrière les cavités coxales II longues de 0,05-0,07 $\mathrm{mm}$, celles du premier sternite apparent longues de 0,03-0,04 $\mathrm{mm}$ et parallèles au bord basal du segment. Edéage long de 0,46-0,53 mm. Partie distale du lobe médian assez déclinée et arrondie à l'apex. Sac interne orné d'une pièce chitinisée plate située dans la moitié apicale, ses membranes finement épineuses dans la moitié basale. Paramères grêles et sinués, bien dépassant l'apex du lobe médian.

\section{Scaphisoma novaecaledonicum Löbl}

Scaphisoma novaecaledonicum Löbl, 1977b: 818, fig. 1, 2; holotype ô: Mt. Koghis; BisHop.

Matériel étudié (11 ex.): la série de types recueillis au Mt. Koghis.

Longueur 1,4-1,7 mm. Tête et pronotum d'un brun rougeâtre plus ou moins foncés, élytres entièrement d'un brun rougeâtre clair. Articles antennaires III à V courts, IV relativement très court, un peu plus long que III, V à peu près de même longueur que III et IV réunis, nettement plus large que IV, VI très grand, plus long que III à V réunis et plus large que V. Pas de microsculpture sur le pronotum. Elytres avec les stries suturales courbées en avant et prolongées le long du bord basal vers le cinquième externe de ıa largeur basale; ponctuation discale sur la base aussi fine ou presque aussi fine que celle du pronotum, progressivement plus grosse vers l'apex. Aires derrière les cavités coxales II longues de $0,04 \mathrm{~mm}$, celles du premier sternite apparent convexes longues de 0,06-0,07 $\mathrm{mm}$. Edéage long de 0,32 $\mathrm{mm}$. Lobe médian assez grêle, sa partie distale à peine déclinée, courbée, atténuée en pointe à l'apex. Sac interne orné de longues épines membraneuses et d'une petite pièce basale un peu plus chitinisée. Paramères grêles et presque droits, dépassant nettement l'apex du lobe médian.

\section{Scaphisoma nanulum Löbl}

Scaphisoma nanulum Löbl, 1973: 314, fig. 9, 10; holotype đ̊: Tiouandé; FrANZ.

Matériel étudié (10 ex.): les 4 types de Tiouandé (près d'Hienghène) et de Table Unio; Mt. Koghis 700 m, 9.X.1978, G. Kuschel, tamisage de feuilles mortes dans la forêt ombrophile, sur la crête, 2 ex.; Rivière Bleue, 10.X.1978, G. Kuschel, tamisage de feuilles mortes et de bois pourri près du pont, 1 ex.; Mt. Rembai 800 m, 21.X.1978, G. Kuschel, tamisage de débris végétaux et de bois pourri, 3 ex. 
Longueur 1,0-1,15 mm. Corps entièrement d'un brun rougeâtre plus ou moins clair. Antennes courtes, article IV assez grêle, à peine deux fois plus long que large; V et VI de même longueur, un peu plus longs que IV, V légèrement plus large, VI nettement plus large que IV. Pronotum sans microsculpture, pourvu d'une ponctuation extrêmement fine. Elytres avec les stries suturales prolongées le long de la base, terminées entre le tiers interne et le milieu de leur largeur basale (vue dorsale); ponctuation discale aussi fine ou à peine plus distincte que celle du pronotum. Aires derrière les cavités coxales II très étroites, longues de $0,02 \mathrm{~mm}$, avec d'assez gros points marginaux. Ponctuation du métasternum partout extrêmement fine, sauf sur le bord antérieur latéral orné de quelques gros points. Aires coxales du premier sternite apparent très étroites, longues de 0,02 $\mathrm{mm}$. Edéage long de 0,23-0,31 mm, grêle. Capsule basale du lobe médian très petite, prolongée par une longue et forte apophyse ventro-distale courbée au niveau de la base des paramères; partie distale du lobe médian longue, régulièrement courbée et progressivement atténuée en pointe. Paramères avec le bord externe légèrement sinué en vue dorsale, un peu élargis dans leur moitié apicale, non ou un peu dépassant l'apex du lobe médian. Sac interne membraneux simple.

\section{Scaphisoma pallens sp. $\mathrm{n}$.}

Holotype đ̊: Mt. Koghis 500 m, 7.X.1978, G. Kuschel, sur un tronc pourri; NZAC. Paratypes: comme holotype 1 \%; Mt. Koghis 550 m, 7.X.1978, G. Kuschel, tamisage de feuilles mortes et de bois pourri sur un terrain plat, 1 . ; Mt. Koghis 650 m, 12.X.1978, J. S. Dugdale, sur un tronc avec champignons, 1 §̊; NZAC, MG.

Longueur 1,2-1,25 mm. Corps peu convexe. Téguments légèrement transparents, entièrement d'un ocre jaunâtre. Antennes et pattes à peine plus claires que le corps. Rapport des longueurs des articles antennaires: III 4 : IV 4 : V 8 : VI 14 : VII20: VIII 13 : IX 18 : X 18 : XI 30 (holotype); article IV très petit, de même longueur ou un peu plus long que III, légèrement plus long que large; $\mathrm{V}$ deux fois plus long que large et un peu plus large que IV; VI 3 fois plus long que large, nettement plus large que V; VII 2,5 fois plus long que large; VIII à peu près 2 fois plus long que large, nettement plus large que VI; XI presque 4 fois plus long que large. Pronotum à la base large de 0,73-0,78 mm, peu rétréci en avant; côtés légèrement arrondis dans les deux tiers apicaux, droits dans le tiers basal; carènes latérales visibles en vue dorsale dans le tiers basal ou sur toute la longueur du pronotum; ponctuation dense et très fine, peu distincte au grossissement $\times 50$; pas de microsculpture. Extrémité du scutellum visible. Elytres longs sur la suture de 0,75-0,78 mm (longueur maximum 0,85-0,89 mm); largeur maximum $(0,85-0,88 \mathrm{~mm})$ situés juste devant le tiers médian de la longueur latérale; bord latéral nettement arrondi dans la moitié basale, presque rectiligne entre le milieu et l'apex; bord apical légèrement convexe; angles apicaux internes situés au même niveau que les angles externes; bord sutural non relevé; espace entre la suture et les stries suturales plat, très finement ponctué; stries suturales assez profondes sauf vers la base où elles sont superficielles, très légèrement courbées à côté du lobe basal du pronotum, pas prolongées le long de la base; ponctuation discale très fine, mais plus distincte que celle du pronotum. Pygidium orné d'une microsculpture formée de points, ponctuation dense et très fine. Mésépimères un peu plus courts que la distance entre eux et les hanches II. Métasternum légèrement bombé au milieu, avec deux dépressions médio-apicales superficielles; ponctuation partout dense et très fine. Aires derrière les cavités coxales II longues de 0,05-0,06 mm, avec des points marginaux très fins. Métépisternes bombés, larges en arrière de $0,08 \mathrm{~mm}$, rétrécis en avant, leur suture interne concave. Sternites 

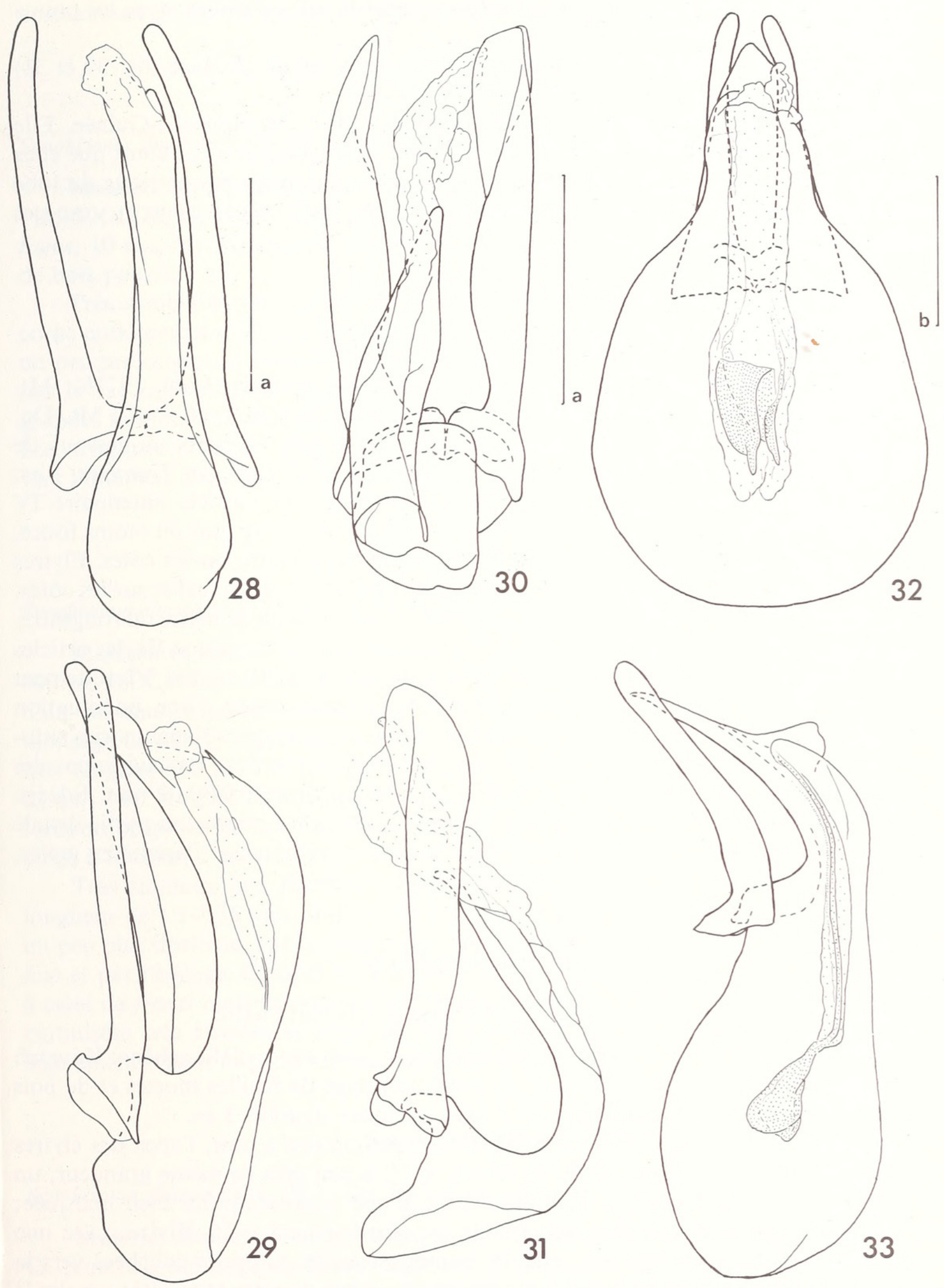

FIG. 28 à 33.

Edéages chez Scaphisoma. 28 et 29. S. funebre sp. n.; 30 et 31. S. pallens sp. n.; 32 et 33. S. variabile sp. $\mathrm{n}$. Echelle a $=0,1 \mathrm{~mm} ; \mathrm{b}=0,2 \mathrm{~mm}$. 
ornés d'une microsculpture formée de points. Ponctuation du premier sternite apparent dense et très fine; aires postcoxales longues de 0,09-0,10 $\mathrm{mm}$, arrondies, avec les points marginaux assez fins. Tibias grêles et droits.

Mâle. Articles 1 à 3 des tarses antérieurs nettements élargis. Edéage (fig. 30 et 31) long de 0,24-0,26 $\mathrm{mm}$

Cette espèce peut être placée près de dissimile Löbl de la Nouvelle-Guinée. Elle en diffère notamment par l'article antennaire IV minuscule (presque aussi court que chez eximium) et par le sac interne entièrement membraneux. La forme asymétrique du lobe médian et les larges paramères peu chitinisées indiquent l'affinité de pallens aux espèces du groupe unicolor.

\section{Scaphisoma eximium Löbl}

Scaphisoma eximium Löbl, 1969: 3, fig.4-7; holotype ô: St. Louis; MG.

Matériel étudié (57 ex.): les types de St. Louis; Mt. Koghis (Löbl, 1977b); Mt. Koghis, 500-550 m, 9.X.1978, J. S. Dugdale, en fauchant dans la forêt, 7 ex.; Mt. Do, sommet 1020 m, 22.X.1978, G. Kuschel, tamisage de débris végétaux au-dessous de Araucaria biramulosa, 1 ex.; Mt. Mou 600 m, 2.XI.1978, J. C. Watt, sur Trametes, 1 ex.

Espèce remarquable par ses palpes maxillaires trapus et l'article antennaire IV extrêmement petit. Longueur 1,5-1,9 mm. Tête d'un brun rougeâtre plus ou moins foncé, pronotum entièrement foncé comme la tête ou plus clair à jaunâtre sur les côtés. Elytres généralement d'un brun foncé à noirâtre sur la base, devant le bord apical et sur les côtés, la zone latérale foncée élargie à l'intérieur au milieu; reste du disque jaunâtre ou rougeâtre. Articles antennaires III et IV minuscules, IV généralement plus court que III, les articles suivants larges mais relativement courts, V plus long que III et IV réunis, VI nettement plus grand que V. Pronotum sans microsculpture. Elytres ornés d'une ponctuation discale très fine sur la base, progressivement plus dense et grosse vers l'apex; stries suturales terminées à la base à côté du lobe du pronotum. Aires derrière les cavités coxales II longues de 0,04-0,05 mm, celles du premier sternite apparent 0,07-0,09 mm. Edéage long de 0,41-0,47 mm. Lobe médian avec la capsule basale assez grande, sa partie distale fortement déclinée et atténuée en pointe. Sac interne membraneux. Paramères grêles, peu courbés, dépassant bien l'apex du lobe médian.

\section{Scaphisoma franzi Löbl}

Scaphisoma franzi Löbl, 1973: 323, fig. 21, 22; holotype §̊: Tindou; Franz.

Matériel étudié (22 ex.): la série typique trouvée à Tindou près d'Hienghène; Kavatch près d'Hienghène, 450 m, 28.X.1978, G. Kuschel, tamisage de feuilles mortes et de bois pourri dans une forêt ombrophile exposée, avec quelques Agathis, 3 ex.

Longueur 1,1-1,2 mm. Corps convexe, d'un brun noirâtre à noir, l'apex des élytres brun. Article antennaire IV très grêle, articles V et VI à peu près de même grandeur, un peu plus longs et larges que IV. Pronotum orné d'une microsculpture bien marquée; sa ponctuation éparse et très fine, peu visible au grossissement $\times 25$. Elytres avec une ponctuation presque aussi fine que celle du pronotum; stries suturales courbées vers la base et terminées à côté du lobe basal du pronotum. Aires derrière les cavités coxales II très étroites, longues de $0,02 \mathrm{~mm}$, avec une rangée de points marginaux assez gros, prolongés sur le bord du métasternum vers les mésépisternes. Edéage long de 0,48-0,52 mm. La partie apicale du lobe médian à peu près aussi longue que la capsule basale, atténuée en pointe peu courbée. Sac interne membraneux, dépourvu d'épines ou de denticules, 
orné d'une pièce médiane plus chitinisée. Paramères grêles et presque droits, dépassant apicalement un peu la pointe du lobe médian.

\section{Scaphisoma simulans Löbl}

Scaphisoma simulans Löbl, 1973: 324, fig. 23, 24; holotype đ̊: Pic du Pin; Franz.

Matériel étudié (29 ex.): les types du Pic du Pin et du Col de Mouirange; Pic du Pin, 6.X.1978, G. Kuschel, tamisage de feuilles mortes dans un ravin peuplé de Nothofagus, 10 ex., Mt. Koghis 550 m, 7.X.1978, G. Kuschel, tamisage de feuilles mortes et de bois pourri, 1 ex.

Très semblable par sa morphologie générale au franzi. Coloration assez variable, corps entièrement d'un brun rougeâtre à noirâtre. Les stries suturales des élytres parfois un peu prolongées le long de la base. Edéage long de 0,50-0,57 $\mathrm{mm}$, très caractéristique par le sac interne muni de nombreuses épines divisées dans la partie basale en deux grandes touffes. Capsule basale très grande, partie apicale du lobe médian courte, recourbée, tronquée à l'apex. Paramères grêles, dépassant un peu le lobe médian et courbés vers l'intérieur dans leur moitié apicale.

\section{Scaphisoma consimile Löbl}

Scaphisoma consimile Löbl, 1973: 326, fig. 25, 26; holotype đ̊: Mt. Koghis; Franz.

Matériel étudié (49 ex.): les types du Mt. Koghis et du Pic du Pin; Col d'Amieu, 400 m, 18.X.1978, G. Kuschel, tamisage de débris végétaux et de bois pourri au bord d'un ruisseau, avec Araucaria, sec; 4 ex.; Mt. Koghis, 550 m, 5.X.1978, G. Kuschel, tamisage de débris végétaux dans la zone entre la forêt ombrophile et le maquis, 1 ex.; Mt. Koghis, 550 m, 7.X.1978, G. Kuschel, tamisage de feuilles mortes sur une pente, 32 ex.; Mt. Koghis 700 m, 9.X.1978, G. Kuschel, tamisage de feuilles mortes dans la forêt ombrophile sur la crête, 3 ex.; Mt. Koghis, 500 m, 5-9.X.1978, J. C. Watt, dans le piège à tomber, 1 ex.

Très similaire aux franzi et simulans, parfois d'une taille légèrement plus grande: longueur de 1,1-1,35 mm. Il diffère de ces deux espèces par la ponctuation du pronotum un peu plus distincte, par la ponctuation sur la partie apicale des élytres nettement moins fine et par l'édéage. Celui-ci long de 0,45-0,52 mm, avec le lobe médian assez semblable à celui de franzi mais sa pointe courbée ventralement, le sac interne orné de deux pièces chitinisées très étroites et muni de fins denticules membraneux; les paramères grêles, légèrement sinués et progressivement atténués vers l'apex.

\section{Scaphisoma viduum Löbl}

Scaphisoma viduum Löbl, 1973: 326, fig. 27, 28; holotype đ̊: Mt. Koghis; Franz.

Matériel étudié (15 ex.): les types du Mt. Koghis; Mt. Koghis 550 m, 5.X.1978, G. Kuschel, tamisage de débris végétaux dans la zone entre la forêt ombrophile et le maquis, 1 ex.; Mt. Koghis 550 m, 5.X.1978, G. Kuschel, tamisage de débris végétaux et de bois pourri dans la forêt ombrophile près du lit d'un cours d'eau peu profond, 1 ex.; Mt Koghis 550 m, 7.X.1978, G. Kuschel, tamisage de feuilles mortes et de bois pourri sur un terrain plat, 1 ex. Yahoué, 11.X.1978, G. Kuschel, tamisage de feuilles mortes et de bois pourri avec Syzygium et Sapindaceae, sec, 1 ex. 
Très semblable aux trois espèces précédentes, mais d'une taille plus grande (longueur 1,4-1,55 mm). Il diffère notamment par la ponctuation du pronotum nettement plus dense et grosse près du bord antérieur. En outre, la ponctuation des élytres est plus distincte que chez consimile, surtout dans le quart ou tiers apical où elle est relativement grosse et assez dense. Edéage long de 0,56-0,60 $\mathrm{mm}$. Lobe médian avec la capsule basale d'une grandeur moyenne, la partie apicale longue, réguliêrement courbée et atténuée en pointe. Sac interne avec une longue pièce basale fortement chitinisée bifurquée dans sa moitié proximale. Paramères en vue dorsale atténués de la base vers le milieu, courbés vers la ligne médiane à l'apex.

\section{Scaphisoma reticulatum Löbl}

Scaphisoma reticulatum Löbl, 1973: 327, fig. 29, 30; holotype đే; Pic du Pin; Franz.

Matériel étudié (71 ex.) : la série typique du Pic du Pin et du Mt. Koghis; Mt. Koghis 550 m, 7.X.1978, G. Kuschel, tamisage de feuilles mortes sur une pente, 1 ex.; Mt. Koghis 700 m, 9.X.1978, G. Kuschel, tamisage de feuilles mortes dans la forêt ombrophile, sur une pente raide, 4 ex.; Pic du Pin, 6.X.1978, G. Kuschel, tamisage de feuilles mortes dans un ravin peuplé de Nothofagus, 1 ex.; Rivière Bleue, 10.X.1978, G. Kuschel, tamisage de feuilles mortes et de bois pourri au-dessous de Nothofagus codonandra, 3 ex.; Col d'Amieu 400 m, 18.X.1978, G. Kuschel, tamisage de feuilles mortes et de bois pourri au bord d'un cours d'eau, sur la côte rocailleuse, avec Araucaria, 1 ex.; Pic d'Amoa près de Poindimié 450 m, 26.X.1978, G. Kuschel, tamisage de débris végétaux dans la forêt ombrophile, 1 ex; les mêmes données mais à $500 \mathrm{~m}$, dans la forêt ombrophile avec Nothofagus aequilateralis et Agathis, 3 ex.

Longueur 1,35-1,5 mm. Par sa taille et par sa morphologie générale très similaire à viduum, en diffère par la ponctuation de la face dorsale presque régulièrement éparse et très fine, semblable à celle de franzi. La microsculpture du pronotum assez variable mais toujours bien distincte. Edéage long de 0,43-0,47 mm. Lobe médian caractérisé par deux saillies ventrales situées au-dessus de la base des paramères; capsule basale grande, la partie distale légèrement déclinée et atténuée en pointe, paramères presque droits, dépassant nettement l'apex du lobe médian. Sac interne avec des membranes munies de nombreux denticules très fins et petits et orné d'une minuscule pièce plus chitinisée située dans sa partie apicale (pas observées sur la série typique).

\section{Scaphisoma alternans Löbl}

Scaphisoma alternans Löbl, 1973: 319, fig. 15, 16; holotype ô: Col de Hau; Franz.

Matériel étudié (7 ex.): les types du Col de Hau; Col d'Amieu (Löвl 1977b).

Longueur 1,15-1,2 mm. Très semblable à franzi, n'en diffère que par la microsculpture du pronotum plus fine et par l'édéage. Celui-ci long de 0,62 $\mathrm{mm}$, son lobe médian plus épais derrière le niveau de la base des paramères, dans le quart apical fortement décliné et atténué en pointe, orné de quelques petits tubercules sur la face ventrale. Sac interne démuni de pièces chitinisées, avec une dense rangée de denticules pliés dans sa moitié basale et de nombreuses épines extrêmement fines dans sa partie apicale. Paramères grêles, en vue latérale légèrement sinués, en vue dorsale atténués derrière le tiers basal, courbés à l'apex vers la ligne médiane. 


\section{Scaphisoma filium Löbl}

Scaphisoma filium Löbl, 1973: 321, fig. 17, 18; holotype đ̊: Table Unio; Franz.

Matériel étudié ( 5 ex.): holotype de Table Unio; Pic d'Amoa près de Poindimié 450 m, 26.X.1978, G. Kuschel, tamisage de débris végétaux dans la forêt ombrophile, 4 ex.

Longueur 1,2-1,25 $\mathrm{mm}$. Les caractères externes comme chez alternans, la microsculpture du pronotum parfois encore plus fine et à peine visible au grossissement $\times 100$. Edéage long de 0,39-0,42 mm, très grêle. Lobe médian assez plat, progressivement atténué, avec l'apex en pointe brusquement courbé. Sac interne long, tout à fait simple, sans pièces chitinisées et sans denticules. Paramères très grêles, légèrement sinués, plus ou moins courbés vers la ligne médiane dans leur quart apical.

\section{Scaphisoma variabile sp. $\mathrm{n}$.}

Holotype đ: Col des Roussettes, 400 m, 26.X.1978, G. Kuschel, tamisage de débris végétaux dans la forêt ombrophile à proximité d'un cours d'eau; NZAC.

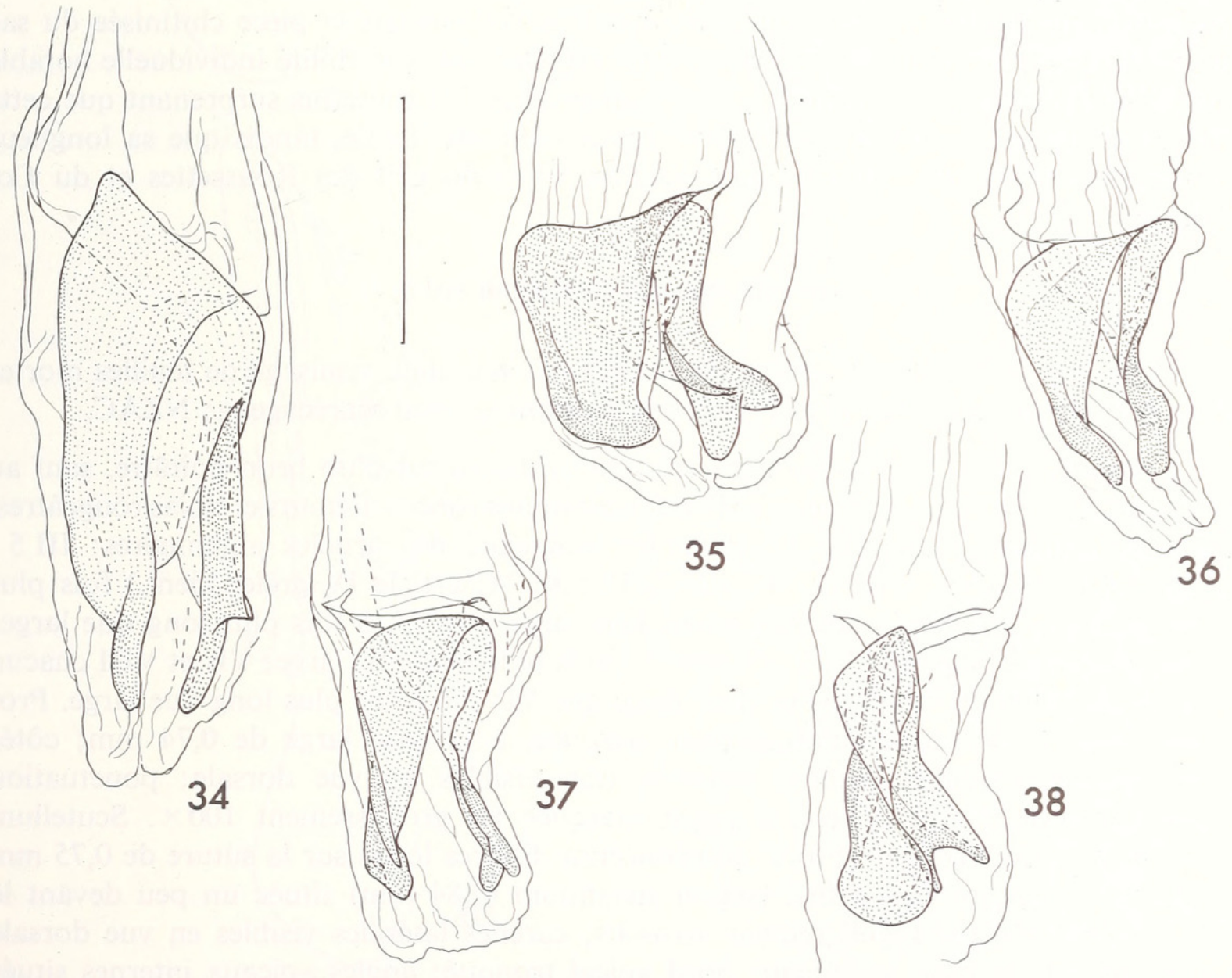

FIG. 34 à 38.

Pièce chitinisée du sac interne chez Scaphisoma variabile sp. n.; 34. exemplaire du Mt. Panié; 35. exemplaire du Col d'Amieu; 36 à 38. exemplaires du Col des Roussettes. Echelle $=0,1 \mathrm{~mm}$. 
Paratypes: comme holotype, $2 \hat{\jmath} 1$ ô; Col des Roussettes 500 m, 31.X.1978, G. Kuschel, tamisage de débris végétaux dans la forêt ombrophile, terrain un peu marécageux, 8 ô 5 ๙ึ; Col d'Amieu 550 m, 15.X.1978, G. Kuschel, tamisage de débris végétaux et de bois pourri dans la forêt ombrophile, ravin avec palmiers, 1 ô; Col d'Amieu 400 m, 18.X.1978, G. Kuschel, tamisage de débris végétaux et de bois pourri à proximité d'un cours d'eau, sur une berge rocailleuse avec Araucaria, sec, 1 \%; Mt. Panié $600 \mathrm{~m}$. 28.X.1978, J. C. Watt, tamisage de feuilles mortes dans la forêt ombrophile, 2 o 3 o; Mt. Panié 250 m, 29.X.1978, G. Kuschel, tamisage de feuilles mortes et de bois pourri dans la forêt ombrophile, 1 đ 1 \%; Mt. Panié, sommet 1638 m, 29.X.1978, J. C. Watt, tamisage de débris végétaux, surtout au-dessous d'Agathis montana, terrain très humide, 2 ๙̃; NZAC, MG.

Avec les mêmes caractères généraux que les autres espèces du groupe reticulatum, mais en moyenne plus grande: longueur 1,5-1,7 mm, pronotum à la base large de 0,891,12 mm; élytres longs sur la suture de 0,86-1,10 (longueur maximum 0,98-1,22 mm), larges de 1,02-1,26 mm. Rapport des longueurs des articles antennaires chez holotype: III 6 : IV 14 : V 16 : VI 16 : VII 20 : VIII 14 : IX 20 : X 20 : XI 27. Microsculpture du pronotum toujours bien développée. Cette espèce paraît particulièrement semblable à viduum, par la ponctuation du pronotum et des élytres bien plus dense et plus grosse sur leurs parties apicales respectives. Elle en diffère cependant très nettement par l'édéage plus robuste (fig. 32 et 33), long de 0,66-0,85 mm, avec des paramères larges à la base, progressivement atténués vers leur tiers apical et surtout par la pièce chitinisée du sac interne large et très distincte, malgré qu'elle présente une variabilité individuelle notable (fig. 34 à 38) affectant sa forme et sa grandeur. Il paraît toutefois surprenant que cette pièce soit longue de $0,19-0,24 \mathrm{~mm}$ chez les 6 ô du Mt. Panié, tandis que sa longueur est comprise entre 0,09 et $0,13 \mathrm{~mm}$ chez les 11 ô du Col des Roussettes et du Col d'Amieu.

\section{Scaphisoma kuschelianum sp. $\mathrm{n}$.}

Holotype ơ: Mt. Panié 250 m, 29.X.1978, G. Kuschel, tamisage de feuilles mortes et de bois pourri dans la forêt ombrophile, endroit un peu marécageux; NZAC.

Longueur 1,15 mm. Corps assez convexe, entièrement d'un brun noirâtre, sauf au bord apical des élytres et de l'apex de l'abdomen moins foncés. Fémurs et tibias rougeâtres, tarses et antennes jaunâtres. Rapport des longueurs des articles antennaires: III 5 : IV 10 : VI 12: VII 18 : VIII 12 : IX $18:$ X 19 : XI 24; article IV grêle, bien 3 fois plus long que large; IV grêle mais nettement plus large que IV, 3 fois plus long que large; VI un peu moins large que V, à peu près 3,5 fois plus long que large; VII et VIII chacun 3,5 fois plus long que large, VIII aussi large que VI; XI 4 fois plus long que large. Pronotum orné d'une microsculpture bien marquée, à la base large de $0,74 \mathrm{~mm}$; côtés régulièrement arrondis; carènes latérales non visibles en vue dorsale; ponctuation presque effacée et très éparse, à peine marquée au grossissement $100 \times$. Scutellum complètement couvert par le lobe du pronotum. Elytres longs sur la suture de $0,75 \mathrm{~mm}$ (longueur maximum $0,80 \mathrm{~mm})$; largeur maximum $(0,84 \mathrm{~mm})$ située un peu devant le milieu; côtés presque régulièrement arrondis; carènes latérales visibles en vue dorsale à proximité de la base seulement; bord apical tronqué; angles apicaux internes situés un peu derrière le niveau des angles externes; bord sutural non relevé; stries suturales assez profondes, courbées à côté du lobe du pronotum mais pas prolongées le long de la base; espace entre la suture et la strie suturale plat, orné d'une rangée de points très fins; ponctuation discale éparse, très fine et superficielle sauf vers l'apex où elle est bien 

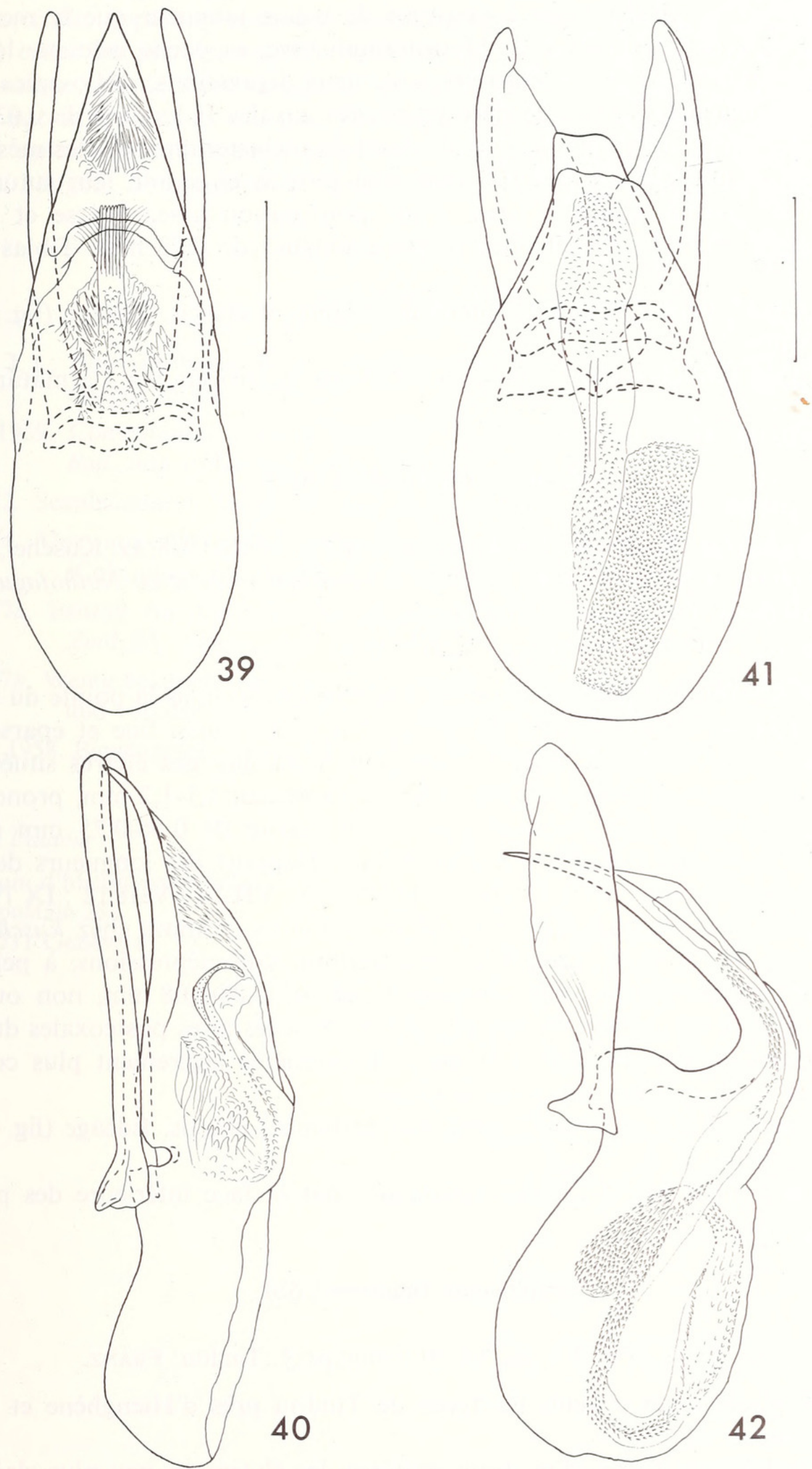

FIG. 39 à 42.

Edéages chez Scaphisoma;

39 et 40. S. kuschelianum sp. n.; 41 et $42 . S$. excellens sp. n. Echelle $=0,1 \mathrm{~mm}$. 
distincte. Pygidium orné d'une microsculpture formée de points; ponctuation assez dense mais très fine. Mésépimères à peu près de même longueur que la moitié de la distance entre eux et les hanches II. Métasternum lisse, sa partie médiane légèrement bombée en avant, presque plate en arrière, avec deux dépressions médio-apicales superficielles à peine marquées. Aires derrière les cavités coxales II longues de $0,03 \mathrm{~mm}$; les gros points marginaux prolongés le long du bord du métasternum vers les mésépimères. Métépisternes bombés, larges de $0,05 \mathrm{~mm}$, non rétrécis en avant, leur suture interne droite. Premier sternite apparent orné d'une ponctuation assez éparse et très fine; aires postcoxales extrêmement étroites, à peine longues de $0,02 \mathrm{~mm}$. Tibias grêles et droits.

Mâle. Articles 1 à 3 des tarses antérieurs nettement élargis. Edéage (fig. 39 et 40) long de $0,48 \mathrm{~mm}$.

Cette espèce est bien caractérisée par l'édéage et surtout par l'armature du sac interne.

\section{Scaphisoma excellens sp. $n$.}

Holotype $\sigma^{\wedge}$ : Pic d'Amoa près de Poindimié, 500 m, 26.X.1978, G. Kuschel, tamisage de débris végétaux et de bois pourri dans la forêt ombrophile avec Nothofagus aequilateralis et Agathis; NZAC.

Paratypes: 2 ô comme holotype; NZAC, MG.

Les mêmes caractères généraux que chez kuschelianum, mais la pointe du scutellum découverte, la ponctuation discale des élytres à peu près aussi fine et éparse dans la partie apicale qu'au milieu du disque, la largeur maximum des élytres située plus en avant, dans le tiers basal de la longueur latérale. Longueur 1,1-1,2 $\mathrm{mm}$, pronotum à la base large de 0,69-0,78 mm, élytres longs sur la suture de 0,66-0,75 $\mathrm{mm}$ (longueur maximum 0,75-0,85 mm), larges de 0,79-0,88 $\mathrm{mm}$. Rapport des longueurs des articles antennaires chez holotype: III 5 : IV 10 : V 13 : VI 15 : VII 18 : VIII 12 : IX 19 : X 19 : XI 28; antennes 'chez les paratypes un peu plus courtes (comme chez kuschelianum); les articles V et VI de même longueur. Métasternum sans dépressions, à peine aplati dans sa partie médio-apicale. Métépisternes larges de 0,06-0,08 $\mathrm{mm}$, non ou un peu rétrécis en avant. Aires derrière les cavités coxales II et les aires postcoxales du premier sternite apparent longues de 0,02-0,03 $\mathrm{mm}$. Mésépimères légèrement plus courts que la moitié de la distance entre eux et les hanches II.

Mâle. Articles 1 à 3 aux tarses antérieurs nettement élargis. Edéage (fig. 41 et 42) long de $0,41-0,45 \mathrm{~mm}$.

Cette espèce peut être facilement distinguée par la face intérieure des paramères élargie et très fine.

\section{Scaphisoma rugosum Löbl}

Scaphisoma rugosum Löbl, 1973: 321, fig. 19, 20; holotype ơ : Tindou; Franz.

Matériel étudié (3 ex.): seuls les types de Tindou près d'Hienghène et de Table Unio sont connus.

Longueur 1,5 mm. Corps d'un brun noirâtre, les élytres un peu plus clairs que le pronotum ou la tête. Article antennaire IV très courts mais nettement plus long que III; V grêle, plus long que III et IV réunis, VI plus court et plus large que V. Pronotum orné d'une microsculpture bien marquée. Elytres avec les stries suturales prolongées le long de la base vers le tiers externe de la largeur basale; ponctuation discale régulièrement 
dense et nettement plus grosse que celle du pronotum. Côtés du métasternum et du premier sternite apparent rugueux. Aires derrière les cavités coxales II longues de $0,05 \mathrm{~mm}$, celles du premier sternite $0,07 \mathrm{~mm}$. Edéage long de 0,44 $\mathrm{mm}$. Lobe médian avec une assez grande capsule basale, sa partie distale légèrement courbée et arrondi à l'apex. Sac interne avec une pièce chitinisée bifurquée située dans sa moitié basale; la moitié apicale membraneuse, munie de nombreuses épines situées dans sa partie inférieure, rangées obliquement à verticalement. Paramères grêles et sibués.

\section{BIBLIOGRAPHIE}

Gressitt, J. L. 1961. Problems in the Zoogeography of Pacific and Antarctic Insects. Pacif. Insects Monogr. 2: 1-94.

LöBL, I. 1969. Contribution à la connaissance des Scaphisomini de la Nouvelle-Calédonie. Bull. Inst. r. Sci. nat. Belg. 45: 1-4.

- 1973. Scaphidiidae (Coleoptera) von Neukaledonien. Archs Sci., Genève, 25: 309-334.

- 1974. Une nouvelle espèce du genre Scaphisoma Leach de la Nouvelle-Calédonie (Coleoptera, Scaphidiidae). Revue suisse Zool. 81: 405-407.

- 1977a. Beitrag zur Kenntnis der Scaphidiidae (Coleoptera) Australiens. Revue suisse Zool. 84: 3-69.

- 1977b. Wenig bekannte und neue Scaphidiidae (Coleoptera) von Neukaledonien, Samoa und von den Fidschiinseln. Revue suisse Zool. 84: 817-829.

Solem, A. 1958. Biogeography of the New Hebrides. Nature 181: 1253-1255.

Adresse de l'auteur :

Muséum d'histoire naturelle

Case postale 284

CH-1211 Genève 6

Suisse 


\section{$2 \mathrm{BHL}$ Biodiversity Heritage Library}

Löbl, I. 1981. "Les Scaphidiidae (Coleoptera) de la Nouvelle-Calédonie." Revue suisse de zoologie 88, 347-379. https://doi.org/10.5962/bhl.part.82380.

View This Item Online: https://www.biodiversitylibrary.org/item/128869

DOI: https://doi.org/10.5962/bhl.part.82380

Permalink: https://www.biodiversitylibrary.org/partpdf/82380

\section{Holding Institution}

Smithsonian Libraries

\section{Sponsored by}

Biodiversity Heritage Library

\section{Copyright \& Reuse}

Copyright Status: In Copyright. Digitized with the permission of the rights holder.

Rights Holder: Muséum d'histoire naturelle - Ville de Genève License: http://creativecommons.org/licenses/by-nc-sa/3.0/

Rights: https://www.biodiversitylibrary.org/permissions/

This document was created from content at the Biodiversity Heritage Library, the world's largest open access digital library for biodiversity literature and archives. Visit BHL at https://www.biodiversitylibrary.org. 